\title{
PREDICTING QUALITY OF LIFE BASED ON HUMOR STYLE
}

\author{
A Thesis \\ presented to \\ the Faculty of California State Polytechnic University, \\ San Luis Obispo
}

In Partial Fulfillment

of the Requirements for the Degree

Master of Science in Psychology

by

Zachary M. Kasow

May 2012 
(C) 2012

Zachary M. Kasow

ALL RIGHTS RESERVED 


\section{ABSTRACT \\ PREDICTING QUALITY OF LIFE BASED ON HUMOR STYLE}

\section{Zachary M. Kasow}

Humor is a multifaceted construct commonly used in daily life. For centuries philosophers, healers, and religious figures have extolled humor as the "best medicine" for both the body and the mind. Recent research has shown humor can be adaptive or maladaptive (i.e., contribute to or subtract from well-being; Martin, Puhlik-Doris, Larsen, Gray, \& Weir, 2003). Empirical evidence supporting these claims for humor and physical health has been inconsistent; however, new evidence suggests there may indeed be a connection (Martin, 2001; Martin et al., 2003). At the same time, previous research has consistently supported the notion that using humor is related to psychological health. Findings such as these have implications beyond one's physical and mental health; these findings also have implications on one's quality of life.

Much like the notion of humor, quality of life is a difficult concept to define succinctly. Quality of life contains several domains including physical health, mental health, social status, and environmental elements (Skevington, Lotfy, \& O'Connell, 2004). Previous research has suggested a link between humor and quality of life.

Adaptive humor is positively correlated with indicators of psychological health, e.g. selfesteem (e.g., Stieger, Formann, \& Burger, 2011) while maladaptive humor has been positively correlated with indicators of psychological distress, e.g. depression (Hugelshofer, Kwon, Reff, \& Olson, 2006). To date, researchers have not studied humor and quality of life directly so the primary purpose of this study was to explore how well humor styles predict quality of life.

To explore this predictive relationship, students from an introductory psychology class at a mid-size university were recruited to participate in this study. It was hypothesized that the adaptive humor styles would positively predict quality of life while the maladaptive humor styles would negatively predict quality of life. Stepwise regression models found partial support for the hypotheses. Implications and future directions are discussed.

Keywords: humor styles, quality of life, physical health, mental health 


\section{ACKNOWLEDGEMENTS}

I would like to thank everyone who has made this project move from just an idea conceived last summer to a completed thesis. Thank you Dr. Teramoto Pedrotti for chairing this committee, offering me so much valuable feedback, and for your overall support through this process. Thank you Dr. Lewis for being a sounding board, offering your unique perspective as a clinician, and for your attentive review. Thank you Dr. Laver for sharing your vast statistical knowledge, methodological guidance, and your willingness to explain everything until I understood it. Thank you Dr. Ryan for your support in allowing me to use the Sona system for participant recruitment and for allowing me to recruit additional volunteers once I reached my first quota. Thank you Adryan Caron for your grammatical contributions and undying support and love throughout this project. Thank you Jenna McKay for offering your editing prowess. 


\section{CHAPTER}

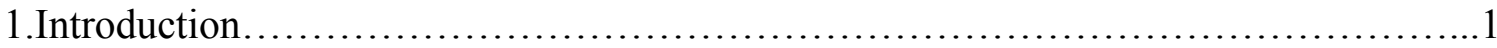

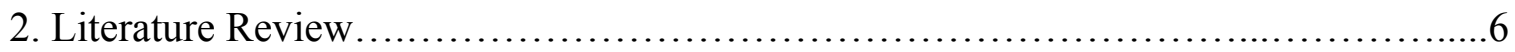

Introduction, 6

Definition of Humor, 6

Theories of Humor, 7

Assessment of Humor, 10

Humor styles, 13

Cultural Considerations of Humor and Humor style, 16

Humor and Physical Health, 20

Humor and Mental Health, 34

Quality of Life, 46

Quality of Life and Humor styles, 48

Conclusion, 49

Reearch Questions, 51

Hypotheses, 51

3. Methods

Participants, 52

Materials, 53

Procedure, 54

Statistical Analyses, 55

4. Results

5. Discussion

Limitations, 61

Future Directions, 62

Conclusion, 64

References. 


\section{List of Tables}

Table

Page

1. Summary of Regression Analyses 
"Two hunters are out in the woods when one of them collapses. He doesn't seem to be breathing and his eyes are glazed. The other guy whips out his phone and calls the emergency services. He gasps, "My friend is dead! What can I do?" The operator says "Calm down. I can help. First, let's make sure he's dead." There is a silence, then a gun shot is heard. Back on the phone, the guy says "OK, now what?"

--Author Unknown, Rated funniest joke by LaughLab

\section{CHAPTER ONE}

\section{Introduction}

Humor is a uniquely human ability with which virtually everyone has experience. An exact definition of humor is elusive because people typically have their own idea of what humor is. If one were to try to explain humor to a race of sentient beings, it might prove to be a tough task, as humor is a difficult concept to define succinctly, and this understanding of what constitutes humor is not universal. In fact, currently there are over 80 theories aimed at explaining humor and the role it plays in our lives (Martin \& Lefcourt, 1984). Some of the most popular theories regarding humor include: superiority theory, psychoanalytic theory, relief theory, and incongruity theory. Superiority theory states humor allows one to feel superior to others (Berger, 1987). Psychoanalytic theory views humor as a defense mechanism (Vaillant 1977, 2000). Relief theory posits that humor is emotional in nature and works to manage the relationship between arousal and

pleasure. Incongruity theory is a cognitive theory, which suggests humor is the result of being presented with an unexpected outcome (e.g., the punch line to a joke).

The superiority and psychoanalytic theories emphasize the social aspects of humor and laid the groundwork for more recent theories and ongoing empirical 
investigation (Martin, 2007). These different theories of humor have led to several ways humor can be conceptualized e.g., a cognitive ability, an aesthetic response, a habitual behavior, a temperament, an attitude, and a coping skill (Martin, Puhlik-Doris, Larsen, Gray, \& Weir, 2003).

Since humor can be conceptualized in so many different ways, researchers have developed different assessments in order to measure sense of humor. Concurrent validity of these measures has been established with other constructs such as intelligence (Feingold \& Mazzella, 1991), personality traits (Cattell \& Luborsky, 1947; Ruch, 1992), and levels of stress (Martin, 1996). These humor assessments were constructed assuming the use of humor is purely adaptive. However, after conducting a review of existing theoretical and clinical literature about humor, Martin et al. (2003) proposed a delineation into four styles of humor: affiliative, self-enhancing, aggressive, and self-defeating. According to Martin and colleagues, the affiliative and self-enhancing humor styles are said to be adaptive because they promote overall wellness, while aggressive and selfdefeating styles are said to be maladaptive humor styles because they do not promote wellness.

The study of humor has taken place primarily in Western societies. One factor that has been shown to impact how an individual defines humor is culture.. Even though Lippa (2007) concluded in a multinational sample that humor is a highly desirable trait for men and women in both same-sex and opposite-sex relationships, research shows humor may not be used the same way in all cultural groups. For example, when humor style scores from several regions of the United States (Southwest Texas, Northwest Texas, Alaska, and Minnesota) were compared to each other, endorsement of each humor 
style was found to be significantly different (Romero, Alsua, Hinrichs, \& Pearson, 2007). Results similar to these have been found for other aspects of culture including race (Thorson, Powell, \& Samuel, 2001), religion (Saroglou, 2004), and gender (Martin et al., 2003). Therefore, any research concerned with the enhancing or detrimental qualities of humor may also not be universal.

Despite culture's impact on its use and definition, humor has been regarded as one of the best medicines for perhaps thousands of years. More recently, Norman Cousins (1979) wrote a book about his experience recovering from a serious medical condition using a self-designed regimen, which included frequently watching humorous television shows and laughing. This anecdotal account set off a renewed interest in the possible health benefits conferred by humor and laughter. Some research has focused on measuring the possible health benefits humor has on physical health, while other research has explored whether a connection exists between humor and mental health.

On one hand the relationship between humor and physical health has been mixed and inconsistent. In his review of the available literature, Martin $(2001,2002,2004)$ concluded that evidence supporting a relationship between humor and physical health is currently lacking. This conclusion was based on several methodological concerns in several of the studies he reviewed and because of the assumption maintained by these researchers that humor is only adaptive (Kuiper \& Martin, 1998; Martin 2001). Research exploring the connection between humor and health using an adaptive/maladaptive framework has been limited; however, there is evidence to support a relationship between humor styles and physical health. 
On the other hand, humor has been shown to consistently correlate with several indicators of mental health. A review of the literature suggests those who use humor feel better emotionally (Martin, 1996). Traditionally, humor has been thought to be only adaptive; however, recently a group of researchers have shown humor can be adaptive or maladaptive (Martin et al., 2003). Adaptive humor is affirming to the self and other, while maladaptive humor is harmful to the self and others. Research has also demonstrated that higher endorsement of adaptive humor usually predicts less shyness (Hampes, 2006), higher social intelligence (Yip \& Martin, 2006), and less vulnerability to depression (Frewen, Brinker, Martin, \& Dozois, 2008). At the same time, higher endorsement of maladaptive humor styles had the opposite relationship with the previously mentioned indicators of mental health. Findings such as these have implications beyond one's mental health but also for one's quality of life.

Much like the notion of humor, quality of life is a difficult concept to define concisely. Quality of life contains several domains including physical health, mental health, social status, and environmental elements. There are perhaps dozens of definitions for quality of life; however, for the purposes of this thesis the definition of quality of life put forth by the World Health Organization (WHO) will be used. The WHO’s Quality of Life Group (1998) defines quality of life as “individuals’ perceptions of their position in life in the context of the culture and value systems in which they live in and in relation to their goals, expectations, standards and concerns" (p. 299). According to this definition, quality of life is comprised of multiple domains including physical health, psychological health, social relationships, and environmental concerns (Skevington, Lotfy, \& O’Connell, 2004). 
Most of the work done with regard to humor and psychological well-being has been correlational in nature (e.g., Kuiper \& McHale, 2009; Stieger, Formann, \& Burger, 2011; Yue, Hao, \& Goldman, 2010). Since causality is impossible to determine when using a correlational design, it is unclear if humor styles create or take away from wellbeing or if well-being creates adaptive or maladaptive styles of coping. The primary purpose of this study is to explore the predictive relationship between humor styles and quality of life. Specifically, this research is meant to determine if quality of life both as a whole, and as separate domains can be significantly predicted by humor style.

To explore this predictive relationship, 100 students from an introductory psychology class at a mid-size university were recruited to respond to self-report measures meant to assess humor style and subjective quality of life. It was hypothesized that the adaptive humor styles would positively predict quality of life, while the maladaptive humor styles would negatively predict quality of life. Stepwise regression models were conducted to determine whether an individual's humor style could significantly predict the individual facets of quality of life as well as overall quality of life. 
"What soap is for the body, laughter is for the soul"
--Yiddish Proverb

\section{CHAPTER TWO}

\section{Literature Review}

\section{Introduction}

Considering the prevalence of humor in daily life, it might seem strange that such an elusive and subjective concept could stand to be classified, reclassified and highly disputed throughout history. Researchers have managed to organize humor into discrete theories, definitions, and defense mechanisms. Previous research has shown a consistent correlation between humor and indicators of psychological health (e.g., positive selfesteem; Martin, 1996). On the other hand, research has not shown such a consistent correlation between humor and indicators of physical health (e.g., increased immune function; Martin, 2001). One potential reason for this relationship not being found is that in previous research humor was conceptualized as only adaptive instead of differentiating between adaptive and maladaptive forms of humor (Martin et al., 2003). This may have influenced the outcomes of these previous studies. The following literature provides support for the notion that humor may predict quality of life.

\section{Definition of Humor}

Perhaps one of the reasons a single understanding of humor has been so elusive is that individuals each have their own definition of humor. Humor may be perceived as involving the telling of funny jokes or stories, as an ability to notice and point out incongruities, being cheerful, or the tendency to see the funny side of things, among other 
definitions (Martin, 2001). In research, humor is not viewed as a single trait (Ruch, 1998); therefore, it is possible to conceptualize humor in many ways (Martin et al., 2003).

For example, some think of humor as a cognitive ability; in this conceptualization humor requires a capacity to understand, create, and reproduce jokes (Feingold \& Mazzella, 1993). Others might view humor as an aesthetic response that suggests appreciation of jokes, cartoons, and laughter along with enjoyment of particular types of humorous materials (Ruch \& Hehl, 1998). Humor can also be conceptualized as a habitual behavior, and in this view attention is paid to how often someone laughs or tells jokes to amuse others (Craik, Lampert, \& Nelson, 1996; Martin \& Lefcourt, 1984). As a temperament trait humor might manifest as habitual cheerfulness (Ruch \& Kohler, 1998). Humor can also be described an attitude such as a positive outlook on the world (Svebak, 1996). Finally, humor can also serve as a coping strategy such as preferring to laugh rather than cry (Lefcourt \& Martin, 1986). These different conceptualizations of humor arise from the various theories of humor.

\section{Theories of Humor}

Just as there multiple conceptualizations of humor, there are also several different theories attempting to explain what humor is and why it is used. Throughout history several theorists have developed and outlined their ideas about what humor is and the role it plays in the lives of those who use it. Currently, it is estimated there are more than 80 different theories of humor (Martin \& Lefcourt, 1984). The theories presented here are some of the most researched theories of humor. Four will be presented: (a) superiority theory, (b) psychoanalytic theory, (c) relief theory, and (d) incongruity theory. 
The superiority theory of humor is perhaps the oldest theory, dating back to the time of Aristotle, and defines humor as it is used in social situations (Lodico, 1998). As the name implies, the purpose of humor according to this theory is to allow someone to feel superior to others (Berger, 1987). This conceptualization of humor can also be interpreted as developing superiority or gaining mastery over one's past failures, shortcomings, or mistakes (Monro, 1967; Robinson, 1983). Being able to laugh at oneself requires control over both self and the environment, which implies superiority (Levine, 1977).

The psychoanalytic theory of humor was first introduced by Freud (1905/1960, 1928). Humor has been considered one of the highest defense mechanisms (Vaillant, 1977, 2000). It is important to note that Freud differentiated types of humor. According to Freud, wit (defined as joke telling to distract the superego) is associated with sexual/aggressive inhibition, comic (defined as nonverbal sources of positive emotions, e.g., the anvil falling on the coyote when he chases the roadrunner) is associated with inhibition, and humor (defined as the perception of something incongruent which allows negative affect to be avoided) is associated with emotional inhibition (Freud, 1905/1960, 1928). Use of humor as a defense mechanism may also promulgate externally. Mulkay (1988) and Oring (1994) both suggest that humor is useful in communicating topics considered taboo by society.

Both the superiority and psychoanalytic theories of humor emphasize social and emotional aspects of humor in an attempt to explain its enjoyable nature (Martin, 2007). Martin argues that even though these theories are no longer very popular in the research world, they introduced concepts still relevant to emerging theories of humor and to 
empirical investigation. The next two theories have a different approach to humor. The relief theory focuses on psychological and physiological arousal while incongruity theory emphasizes the cognitive aspects of humor.

The relief theory of humor (also known as the arousal theory of humor) is psychophysiological in nature. Relief theory was heavily influenced by the $19^{\text {th }}$ century view of the nervous system, the so-called hydraulic theory of nervous energy, which was modeled after a steam engine (Martin, 2007). This theory suggests humor enjoyment can be understood based on an inverted-U function of arousal and pleasure (Berlyne, 1972). Berlyne suggested that either too much or too little arousal is unpleasant. Subsequent research has shown little evidence for an inverted-U function of arousal and pleasure, and instead the relationship appears to be more linear where humor seems to boost arousal (Martin, 2007). According to Martin, this theory of humor lends support to the notion that humor is an interaction between emotion and cognition. This theory has inspired further research (e.g., Ruch, 1997) seeking to determine the physiological basis of humor.

Finally, the incongruity theory of humor is cognitive in nature because it requires one to shift his or her frame of reference unexpectedly to reconcile disparate information (Lefcourt \& Martin, 1986). According to this theory, a joke or situation is funny because of the expectation of one thing and the delivery of another. Incongruity is a common theme in jokes where the punch line comes as a complete surprise (Lodico, 1998). There is some disagreement about the role of resolution of the incongruity. Nerhardt (1976) and Rothbart (1973) argue that incongruity can stand alone to create humor. On the other hand, Suls $(1972,1983)$ argues that humor requires incongruity to be resolved. Research to support incongruity theory continues to this day (Martin, 2007). 
In the process of investigating and trying to find support for these different theories of humor, researchers needed to develop methods for measuring humor. Early research into the superiority and psychoanalytic theories of humor made use of jokes and cartoons and asked participants to rate their funniness (Mindess, Miller, Turek, Bender, \& Corbin, 1985). The jokes and cartoons depicted various levels of aggression or hostility. Instead of the participants' ratings of funniness, relief theory researchers measured sympathetic arousal (i.e. heart rate, cortisol levels, and skin conductance) before and after exposure to humorous materials such as comedy films (e.g. Hubert, Moeller, \& de JongMeyer, 1993). Incongruity theory researchers have returned to the use of jokes and cartoons and asking participants to rate their funniness; however, these researchers manipulated whether incongruity was present (e.g., Deckers, 1993).

\section{Assessment of Humor}

The various researchers experienced, as mentioned previously, that different conceptualizations and theories of humor lend themselves differently to assessment. For example, if a researcher wanted to measure humor as an aesthetic response, he or she may rate participants' ratings of funniness of jokes or the frequency in which participants laughed (Martin \& Lefcourt, 1984). In the last century, researchers have developed, validated, and used several different humor measures while exploring the relationship between humor and other constructs. Since there is no universal conceptualization of humor, there are viable humor measures for each conceptualization of humor.

Many of the earlier humor measures targeted one conceptualization of humor. Many of these early assessments were self-report. One of the earliest humor measurements was the IPAT Humor Test of Personality developed by Cattell and 
Luborsky (1947). This scale includes a total of 104 pairs of short jokes; respondents are instructed to choose which joke is funnier in each pair. Humor-related characteristics are in this way measured across 13 different dimensions which correlate with other measures of personality, e.g., Introversion-Extroversion. From there, a range of other assessments were developed including the 3-Witz Dimension Humor Test (3WD; Ruch, 1992), the Antioch Sense of Humor Inventory (ASHI; Mindess et al.,1985), The Humor Cognition Test (HCT; Feingold \& Mazzella, 1991), the Coping Humor Scale (CHS; Martin \& Lefcourt, 1983), the Humorous Behavior Q-Sort Deck (HBQD; Craik, Lampert \& Nelson,1993, 1996), and the Situational Humorous Response Questionnaire (SHRQ; Martin \& Lefcourt, 1984). These tests each conceptualize humor differently and have all been used in the humor literature.

The IPAT (Cattell \& Luborsky, 1947; Cattell \& Tollefson, 1966), 3WD (Ruch, 1992), and the ASHI (Mindess et al., 1985) were designed to measure humor as an aesthetic response, more specifically the appreciation of humor. These tests are each meant to determine what kind of humor the person taking the test prefers. For example, the 3WD measures the funniness and averseness of three categories of humor: incongruity-resolution humor, nonsense humor, and sexual humor (Ruch, 1992). Once an individual's preference for a type of humor is identified, it can then be correlated to different personality traits (e.g., extraversion). Some studies suggest there are relationships between preference for specific types of humor and personality traits.

Scales like the HCT conceptualize humor as a cognitive ability. Feingold and Mazzella (1991) developed the HCT while investigating the relationship between humor and intelligence. The HCT yields a humor cognition score in addition to two subscores: 
joke knowledge and joke reasoning. These researchers found there is a significant positive correlation between scores on the HCT and other measures of cognitive ability (e.g., SAT/GRE scores).

Assessments conceptualizing humor as a habitual behavior include the HBQD (Craik, Lampert, \& Nelson, 1993, 1996) and the SHRQ (Martin \& Lefcourt, 1984). The purpose of assessments like these is to determine how often one laughs or tells jokes to amuse others. The SHRQ is a short self-report measure, which asks a respondent to indicate the degree to which he or she would laugh if he or she were in a variety of different, everyday life situations (e.g., a waiter spilling a drink on you). Scores on measures like the SHRQ have been positively correlated with indicators of mental health (Martin, 1996).

The conceptualization of humor as a coping strategy has also lead to the development of a humor assessment. Martin and Lefcourt (1983) developed the CHS specifically for a study meant to explore the stress-moderating effects of humor. Since its introduction, it has been used in over 40 published studies aimed at elucidating the relationship between humor and both physical and mental health. The CHS is a short measure with questions asking how likely the respondent is to use humor to deal with life's stresses.

While assessments that conceptualize humor in one way have been useful, researchers began to develop humor assessments meant to measure multiple conceptualizations of humor at once. There are two examples used most widely in the research literature. The first example is the Multidimensional Sense of Humor Scale (MSHS) developed by Thorson and Powell (1991, 1993a). The MSHS is a short, self- 
report measure that assesses four aspects of humor: humor creation for social purposes, coping humor, appreciation of humorous people, and appreciation of humor. The scores from each of these aspects of humor are added to create an overall sense of humor score. Thorson and Powell (1993a) intended the MSHS to be used so humor could be correlated with personality traits and to compare different groups of people with regard to the four aspects of humor measured by the scale. Since its validation, the MSHS has been used for these purposes.

The second example of a humor assessment that measures multiple conceptualizations of humor at once is the Humor Styles Questionnaire (HSQ: Martin et al., 2003). Previous humor assessments all assume that humor is always an adaptive trait. Martin and his colleagues found evidence in previous literature that suggested this assumption might not be true. Their findings indicated humor could serve both adaptive and maladaptive purposes. Therefore, the researchers developed a new humor assessment to distinguish adaptive and maladaptive styles (i.e., types) of humor. This measure, as well as all of the other measures reviewed in this section, was developed and validated in a Western society. As such, the questions asked are Western-focused and may not be as applicable to research participants from a non-Western background.

\section{Humor styles}

Current research has shown that humor is not always adaptive as was once thought. Martin et al. (2003), in a comprehensive review of past theoretical and clinical literature, posited that four distinct humor styles exist: (a) affiliative, (b) self-enhancing, (c) aggressive, and (d) self-defeating. These researchers conceptualize the functions of humor as both intrapsychic and interpersonal. In line with this differentiation, two 
distinctions are made when considering humor style. The first is whether humor is used to enhance the self or one's relationships with others. The other distinction is whether humor is benign/benevolent or potentially detrimental either to the self or others.

In an exploratory factor analysis, Martin and colleagues (2003) found that these four styles appear to be distinct from one another, as is supported by fairly weak correlations between the various scales. As such, individuals may relate to each humor style to one degree or another, however, one humor style will likely stand out as the primary personal humor style. Two of these humor styles (affiliative and self-enhancing) are considered adaptive because they facilitate coping and social bonds, while the other two humor styles (aggressive and self-defeating) are considered maladaptive (Martin et al., 2003).

\section{Affiliative humor}

Affiliative humor was first suggested by Vaillant (1977). Individuals high in affiliative humor tend to use humor to amuse people around them, to facilitate relationships, and to reduce interpersonal tensions (Lefcourt, 2001). Martin et al. (2003) view affiliative humor as relatively benign as well as self-accepting because it affirms the self and others; this quality possibly enhances cohesiveness and attraction. Though an individual high on this dimension may use self-deprecating humor, its purpose is still to put others at ease and to maintain a sense of self-acceptance (Vaillant, 1977).

\section{Self-enhancing humor}

Freud (1928) was the first to describe the self-enhancing style of humor when describing humor in general as a "mature defense mechanism" (p. 4). Individuals high in self-enhancing humor generally have a humorous outlook on life even when they face 
stress or adversity (Kuiper, Martin, \& Olinger, 1993). One high in this dimension can keep themselves in good spirits without reliance on other people. Self-enhancing humor is closely related to coping humor (e.g., telling jokes to cope with life's stresses; Martin, 1996), perspective-taking humor (e.g., telling jokes to place distance between self and an unpleasant experience; Lefcourt et al. 1995), and humor as emotion regulation/coping mechanism (e.g., telling jokes about unpleasant experiences; Dixon, 1980; Martin, Kuiper, Olinger, \& Dance, 1993).

\section{Aggressive humor}

Aggressive humor was originally called disparaging humor by Zillman (1983). Individuals high in aggressive humor tend to put other people down or belittle them using sarcasm, teasing, ridicule, derision, or "put-downs," possibly under the guise of fun (Zillman, 1983). Janes and Olson (2000) suggest that aggressive humor does not have to actively put others down, but can also be an implied threat of ridicule (for example, being present when someone else is being made fun of or ridiculed). Aggressive humor involves a disregard for the impact of one's [humorous] comments on others (Martin et al., 2003). Martin et al. point out there can be an element of impulsiveness that accompanies aggressive humor because of the tendency to blurt things out that may be harmful to others.

\section{Self-defeating humor}

Self-defeating humor was first described by Kubie (1971) when describing the dangers of using humor in psychotherapy. Individuals high in self-defeating humor tend to be excessively self-disparaging (Martin et al., 2003). People high in his domain either allow themselves to be the target of others' jokes or put themselves down for the sake of 
being accepted by others. This type of humor also may be used to repress one's feelings in exchange for the acceptance of others (Kubie, 1971). While individuals using this type of humor may be perceived as witty or amusing, they tend to have an element of emotional neediness, avoidance, and low self-esteem (Fabrizi \& Pollio, 1987).

As outlined above, individuals typically adhere to an either adaptive or maladaptive humor style, with occasional variance depending on the situation (Martin et al., 2003). Not only can these styles convey the speaker's sense of self-acceptance and level of social integration, they may also have a direct effect on mental health, with possible additional benefits for physical health (Martin et al., 2003). Thus, the ways in which one utilizes or perceives humor have implications for overall quality of life. Since these humor styles were developed in a Western society, culture might influence how one utilizes and perceives humor so it is vital to examine how humor is conceptualized crossand multi-culturally.

\section{Cultural Considerations of Humor and Humor Styles}

As mentioned previously, humor is difficult to define among individuals and may not be the same in all cultural groups e.g., different races, ethnicities, or genders. Many of the studies regarding humor have been conducted in Western cultures (e.g., Martin et al., 2003), which may inadvertently set up deficit models with non-Western groups who may conceptualize humor differently. For example, Lippa (2007) found that humor is highly desired by both men and women in same-sex oriented and opposite-sex oriented relationships in 53 nations. Though this suggests that humor may be common, it does not prove that humor is conceptualized the same way universally. 
Martin et al. (2003) examined possible differences in humor styles with regard to gender and age. These researchers found no significant differences between men and women on the adaptive humor styles. However, there was a significant difference between men and women on the maladaptive styles. The results showed that men tend to endorse both the aggressive and the self-defeating humor styles more often than women (Martin et al., 2003). These researchers also found that younger participants scored significantly higher on the affiliative and aggressive humor styles than older participants. When comparing humor styles in the United States and Croatia, Thorson, Brdar, and Powell (1997) found the sample from the United States valued humor creation more than the sample from Croatia. These researchers suggested that differences in language and culture might influence how different groups conceptualize humor. Two studies in particular have demonstrated this difference in conceptualization, one in samples of African Americans and White Americans (Thorson, Powell, \& Samuel, 2001) and another between a sample from Spain and a sample from the United States (CarbeloBaquero, Alonso-Rodriguez, Valero-Garces, \& Thorson, 2006).

Comparing a sample of White university students and another sample of African American university students Thorson et al. (2001) found no significant difference in overall scale scores. However, these researchers did find some differences in how participants responded to certain scale items. In particular, these researchers found White respondents averaged higher scores than African American respondents on a question measuring the ability to use humor to cope with stresses, and another measuring humor appreciation; African American respondents averaged higher scores on a humor creation question. These results could ensue from multiple origins, including lack of conceptual 
equivalence of the construct of humor across these two groups or lack of metric equivalence in differing levels of endorsement between the two groups (Lonner \& Berg, 1986). These researchers concluded culture does indeed influence how humor is used and expressed.

Another group of researchers came to a similar conclusion using cross-cultural samples from the United States and Spain. Unlike Thorson et al. (2001), CarbeloBaquero and colleagues (2006) found significant differences between scale scores. The results from this study indicate that people from the United States place more importance on humor production (e.g., telling jokes) while people from Spain place more emphasis on using humor to cope with difficult life situations (e.g., loss of a job). Though further research is necessary, culture seems to have some impact on the utilization of humor.

There is the potential for various regions of a particular country to have an influence on humor. Comparing four different regions of the United States (Alaska, Southwest Texas, Northwest Texas, and Minnesota) Romero, Alsua, Hinrich, and Pearson (2007) determined there is a significant difference in humor styles across these regions. For example, participants in Minnesota scored significantly higher on affiliative humor than those in southwest Texas. Zelinsky (1992) described ten major cultural regions within the United States based on food, language, accent, music, and traditions. Romero et al. (2007) concluded there are likely significant differences in humor in each of the regions outlined by Zelinsky (1992). Based on this conclusion for example, people who reside in New England compared to those residing in the South are likely to exhibit significant differences in humor. 
Research indicates that religion may also play a role in how humor is perceived. Saroglou (2004) examined how religion influences the use of humor. This researcher was interested in the strength of religiosity/spirituality rather than any particular religion. Results from this study suggest people who score high on religiosity/spirituality tend to use humor less often than those who score lower on religiosity/spirituality measures. Saroglou (2004) concluded being religious implies a different kind of humor.

Since its creation, the HSQ has been translated and used in studies in several countries. Saroglou and Scariot (2002), Kazarian and Martin (2004), Kazarian and Martin (2006), Taher, Kazarian, and Martin (2008), and Chen and Martin (2007) have found that the four-factor structure of humor styles exists in Belgium, Lebanon, and China. While this may address linguistic equivalence (i.e., the measure has been translated appropriately), conceptual equivalence may not occur across cultural groups (Lonner \& Berg, 1986). Along these lines, it is worth noting the aggressive humor style was less distinct in the sample of Lebanese community sample (Taher et al., 2008). Chen and Martin (2007) found similar results in a sample of Chinese students. It is possible that the aggressive humor style may not be as strongly related to mental health in collectivistic cultures compared to individualistic cultures (Chen \& Martin, 2007; Taher et al., 2008). These findings lend support to the notion that language and culture may influence an individual's conceptualization of humor.

The multicultural and cross-cultural research reviewed above suggests that humor is not the same for everyone. One's culture does seem to have an impact on humor. The research conducted to find a relationship between humor, physical health, and mental health has been conducted almost exclusively in Western cultures. With this in mind, it 
is important to remember the findings of the following research cannot be assumed to be universal for all cultures.

\section{Humor and Physical Health}

For much of recorded history, philosophers, religious teachers, and even doctors have recommended humor as "the best medicine" for both the body and the soul.

Norman Cousins' (1979) account of recovering from ankylosing spondylitis (a debilitating form of arthritis) following a self-designed and prescribed regimen of humor, laughter, and other alternative medical approaches revitalized this notion. Media reports of scientific research indicating potential health benefits like lowering blood pressure, lowering stress hormones, improving immunity, improving memory, and exercising the diaphragm help to reinforce this age old adage (Martin, 2002). So far, four theoretical mechanisms that may explain a possible link between humor and physical health have been proposed.

The first theoretical mechanism suggests that laughter brings about physiological changes in the musculoskeletal, cardiovascular, endocrine, immunological, and/or nervous system may have a beneficial effect on health (Martin, 2001). Previous research has found that laughter influences catecholamine and cortisol levels in the body, which may influence immune function (Dantzer \& Mormede, 1995; Hubert et al., 1993). According to this theoretical mechanism, forced or feigned laughter may be beneficial without the presence of humor. The belief that laughter, even without the presence of humor, is beneficial is maintained by practitioners of yogic laughter exercises (Martin, 2001). 
The second theoretical mechanism suggests the connection between physical health and humor is the result of positive emotional states accompanying laughter and humor (Martin, 2001). Previous research has shown that positive emotions (e.g., mirth) may have analgesic effects (Bruehl, Carlson, \& McCubbin, 1993) immune enhancing effects (Valdimarsdottir \& Bovbjerg, 1997) or have an reducing effect on cardiovascular stress resulting from negative emotions (Fredrickson, 1998). Unlike the previous theoretical mechanism, this mechanism suggests that actual laughter is not needed for any benefits to be experienced. Instead, what is required for physical health benefits are the positive emotional states that accompany humor.

The third theoretical mechanism suggests the health benefits of humor are derived from the stress moderating effects of humor (Martin, 2001). In this hypothesis, humor's effect on health is indirect, decreasing stress levels, which then reduce the harmful effects of stress on health. Evidence has shown that stress influences various aspects of health, e.g., suppression of immune system (Adler \& Hillhouse, 1996) and increased risk of infection (Cohen et al., 1998). Personality variables and coping strategies serve to moderate stress through cognitive appraisals and attributions (Martin, 2001).

The fourth and final mechanism also suggests humor's role in health enhancement is indirect (Martin, 2001). According to this mechanism, humor facilitates social support, which boosts health. Humor has been shown to decrease interpersonal conflict and improve social cohesion in peer groups; humor is also correlated with social competency (Martin, 2004). Accordingly, humor can be used to develop social support. Social support may confer stress buffering and health-enhancing effects (Orth-Gomer, 1994). 
Most of the empirical studies attempting to establish a connection between humor and physical health have focused on five areas (Martin, 2001). According to Martin (2001) these five areas are: pain tolerance, blood pressure, immunity, self-reported illness symptoms, and longevity. These studies have been either experimental, correlational, or stress moderating designs.

\section{Humor, Laughter, and Pain}

The research conducted to connect humor and laughter with pain tolerance or threshold was probably inspired by Cousins' (1979) account of the analgesic effects humor and laughter had for him while he was recovering from a painful medical condition. Drawing on his account, several teams of researchers began to study how humor and laughter might influence an individual's pain tolerance or threshold. These researchers used several different methods to measure pain tolerance and threshold (e.g., the squeeze of a blood pressure cuff). According to Martin (2001) these studies have been the most carefully controlled of any of the studies examining the connection between humor and health. However, the results reported have been inconsistent.

Multiple studies have found that humor seems to have an impact on pain tolerance. Using a sample of university students, Hudak, Dale, Hudak, and DeGood (1991) randomly assigned participants to watch either a comedy film or a documentary. Baseline pain thresholds (based on transcutaneous end nerve stimulation) were assessed before participants began watching the video, and smiles and laughter were monitored while participants watched the videos. Participants also filled out the SHRQ. Hudak et al. (1991) found participants in the comedy group had significantly higher pain thresholds than those in the documentary condition. These researchers found no correlation between 
the humor measure and pain threshold. These results suggest that exposure to humorous stimuli might help one tolerate more pain.

Similar results have been found in subsequent research. The influences of mood, behavior, and cognition have also been investigated by Zweyer, Velker, and Ruch (2004). Participants in this study all watched a humorous movie but were assigned to one of three groups. The first group was instructed to get into a cheerful mood without laughing, the second group was told to laugh and smile, while the third group was told to produce humorous commentary for the movie. Pain tolerance was assessed before watching the movie, immediately after watching the movie, and 20 minutes after watching the movie. All three groups experienced an increase in pain tolerance immediately after watching the movie and still experienced the effect 20 minutes after the movie ended. These researchers concluded that humor can increase pain tolerance, and more research needs to be done to determine how long the increased pain tolerance effect lasts.

Despite the aforementioned, not every study has found humor to be associated with increased pain tolerance. For example, one of the first published studies examining the possible connection between pain threshold and humor was conducted by Cogan, Cogan, Waltz, and McCue (1987). These researchers conducted two studies each measuring pain threshold induced by a blood pressure cuff. In both experiments, participants were divided into groups that were randomly assigned to listen to a humor tape, a relaxation tape, a dull narrative, or nothing at all. The results indicated individuals assigned to the comedy condition and relaxation condition had significantly higher pain thresholds than participants in a dull narrative or no treatment condition. Similar results were reported by Nevo, Keinan, and Teshimovsky-Arditi (1993) who assigned a sample 
of undergraduate students to one of three groups. These groups watched a comedy film, a documentary film, or no film. Pain tolerance was assessed using a cold-presser task. These researchers found no difference in pain threshold across all three experimental conditions.

Several researchers have investigated the effects humor has on individual pain tolerance. To date, more studies have shown humor does indeed pain tolerance; however, these results have not been consistent across all studies. Considered together, the results from these studies suggest pain tolerance might be influenced by the use of humor. At this time it is unknown how long these analgesic effects last. The exact mechanism by which humor increases pain tolerance also remains unknown.

\section{Humor, Laughter, and Blood Pressure}

The humor and physical health connection has also been explored by examining how using humor can impact an individual's blood pressure. The presence of high blood pressure can be indicative of other health problems, and can have several adverse effects on one's health by itself; for example, high blood pressure increases one's risk for stroke, heart attack, and renal failure among other life threatening conditions (Guyton \& Hall, 2005). If the use of humor can reduce one's blood pressure, it could prove to be a useful intervention in improving one's health. Some research suggests humor may reduce blood pressure, but these results have not been consistent in the literature.

Some research supports the notion that humor is associated with blood pressure. Lefcourt, Davidson, Prkachin, and Mills (1997) measured blood pressure before using lab stress tasks. These researchers also administered some self-report humor measures. They found females with higher scores on the self-report humor measures showed lower 
systolic blood pressure while the opposite was found for males. Using a self-report of laughter over a three-day period, another group of researchers found results similar to those reported by Lefcourt et al. (1997). Martin and Kuiper (1999) determined there is a correlation between laughter frequency and Type A behavior. Men who reported more laughter in the previous three days also reported more Type A behaviors than those who reported less. On the other hand, women who reported more laughter in the previous three days reported fewer Type A behaviors (Martin \& Kuiper, 1999). Both groups of researchers concluded men may use humor differently than women, i.e., men may be more likely to express humor in a maladaptive way than women. These conclusions have since been supported by research conducted on humor styles.

Not all investigations have yielded results to support a connection between humor and blood pressure. White and Camarena (1989) conducted a study to determine the different effects of several interventions aimed at lowering blood pressure and heart rate toward the promotion of better overall health. These researchers divided participants into the following groups: laughter, relaxation training, and health-education control group. Each group met for an hour and a half per week for six weeks to practice. The laughter group practiced laughing yoga that utilizes laughter that is forced, assuming the body cannot tell the difference between real laughter and fake laughter. The relaxation training group learned techniques like progressive relaxation and breathing meditation. Finally, the health education group learned about topics like exercise and wellness. Results showed there was no difference between the pre-test and post-test scores in the laughter group and there was no significant difference in either heart rate or blood pressure between the laughter group and the health-education control group. These results were 
similar to those obtained in a longitudinal study of Finnish police officers aimed at determining whether sense of humor predicts better physical health (Karkkanen, Kuiper, $\&$ Martin, 2004). These researchers used a sample of Finnish police chiefs. Results indicated sense of humor does not predict levels of physical health or workplace wellbeing.

The research surrounding blood pressure and humor is still not very conclusive. Results from some studies suggest that use of humor by women (and presumably anyone who uses in humor in an adaptive way) can decrease blood pressure. Other studies failed to find results suggesting humor is even related to blood pressure. Subsequent research exploring the connection between humor and mental health has since confirmed the existence of adaptive and maladaptive humor and supports the conclusion that men use more maladaptive humor than women.

\section{Humor, Laughter, and Immunity}

Humor's relationship with immunity has received more attention from researchers interested in the connection between humor and health as evidenced by the higher number of empirical studies. Immunity was measured in different bodily fluids (i.e., saliva or blood) using immunity indicators such as Immunoglobulin A (IgA), T-cell helper-suppressor ratio, blastogenesis, and Natural Killer (NK) cell activity (Martin, 2001). The assumption in this research is if humor does indeed boost physical health, there will be a significant increase in immune markers when an individual uses humor.

One of the earliest studies examining humor and immunity was conducted by Dillon, Minchoff, and Baker (1985). These researchers asked a student sample to watch a 30-minute comedy tape and a 30-minute educational tape in counterbalanced order with 
a 10-minute break in between films. Those who viewed the comedy film experienced a significant increase in immune markers, but there was no pretest to posttest change in the education tape condition. Direct comparisons were not made between the conditions in this experiment, so it is unclear if there is a difference between these two groups. Using the same sample, these researchers compared scores on a coping humor scale and an immune marker found in saliva. They found a positive correlation between use of coping humor and immune function. These results indicate humor increases immune function. Results like this have been replicated several times.

The results of Dillon et al. (1985) kicked off more research exploring the relationship between humor and immune function. Using a sample of new mothers Dillon and Totten (1989) measured immune function. The participants also filled out a coping humor scale within a week of delivery and then again two months after delivery. These researchers found a moderately positive correlation between the immune function and the coping humor scale. In another study, Lefcourt, Davidson-Katz, and Kueneman (1990) performed three experiments examining the effects of exposure to comedy on immunity markers. Participants watched two comedy tapes by two different comedians, and immune markers were measured before and after watching the tapes. Leftcourt et al. found a significant increase in immune markers after watching the comedy tapes. Participants also filled out a coping humor questionnaire and a questionnaire meant to measure situational humor. Lefcourt et al. (1990) found no correlation between these questionnaires and immune function either at baseline or after watching the comedy videos. Combined, these results show exposure to humorous stimuli tends to increase 
immune function. However, the tendency to laugh in a variety of situations does not seem to be related to immune function.

Results mentioned previously all add support to the notion humor increases immune function. Ensuing research has added more weight to this notion. Lambert and Lambert (1995) used a sample of fifth-grade students in their investigation into the connection between humor and physical health. The students were split up into two groups: one group watched 10 minutes of live comedy followed by another 15 minutes of a popular TV show featuring funny home videos, while the other group watched a 10minute lecture about proper hand-washing and a 15-minute tape about the weather. The comedy group showed a significant increase in immune markers. McClelland and Cheriff (1997) conducted a series of three experiments in which participants watched a comedy, a documentary about World War II, or an instructional video about filling out a financial aid form. These researchers found no change in immune markers in either the World War II documentary or the financial aid instructional video groups, while there was a significant change in the comedy film group.

In the above literature, humor groups tend to see significant increases in immune function while non-humor groups do not. This result has been consistent across all research reviewed. Taken together, actually engaging in humor (i.e., watching a funny video and laughing) seems to increase immunity. People who endorse using coping humor more also tend to have better immune function. On the other hand, having a tendency to use humor in a variety of life settings does not correlate with increased immune function. On the surface, these results provide strong evidence that using humor 
can improve health. Martin (2001) presents several methodological concerns that will be discussed later in this section.

\section{Humor, Laughter, and Self-reported illness symptoms}

When researchers have not had access to techniques involving measurement of pain tolerance, blood pressure, or immunity, the self-report of physical illness symptoms has been used. These measures ask about the frequency of various physical symptoms, e.g., aches, pains, and infections. Several studies mentioned previously in this section employed experiments combined with a correlational design; the results reviewed in this section were all correlational. Like some of the other areas of research into the connection between humor and physical health, the results from studies like these are mixed.

Some research suggests scores on some of the measures of humor correlate with self-reported physical symptoms measures. After recruiting a sample consisting of people over the age of 55, Simon (1990) administered the SHRQ and a health perception measure. Results suggest a negative correlation between situational humor assessment and health perception. However, there was no correlation between scores on the coping humor scale and the health perception measure. Other studies have found similar results in other samples, i.e., university students and female business executives. In a sample of undergraduate students, Carroll and Schmidt (1992) obtained results analogous to those of Simon (1990) using a different inventory of physical health problems. Using a sample of female business executives, Fry (1995) found a significant correlation between the scores on the coping humor scale, scores on the situational humor scale, and scores on a self-report measure of symptomatology. Finally, using another sample of university 
students, Ruch and Kohler (1999) measured cheerful composure and correlated these scores with a self-report inventory of bodily complaints. These researchers found a negative significant correlation between these two measures. Considered together, these combined results suggest that the use of humor corresponds to self-reported illness symptoms.

While there have been several studies supporting a relationship between humor and self-reported physical health, several other studies have failed to replicate the results previously mentioned. Using a sample of undergraduate students, Porterfield (1987) found no correlation between coping humor scores, situational humor scores, and scores on a physical symptom checklist. Anderson and Arnoult (1989) found no correlation between humor scale scores, physical illness symptoms, overall wellness, and frequency of insomnia. Labott and Martin (1990), Korotkov and Hannah (1994), and McClelland and Cheriff (1997) all found a similar lack of results in this direction.

As mentioned previously, the notion that humor is the best medicine has existed for a long time. Interested to determine potential reasons for the continuing popularity of this notion, Kuiper and Nicholl (2004) conducted a study to find out. Instead of correlating only physical illness symptoms with a sense of humor scale, these researchers instead examined other health behaviors in addition to physical illness symptoms (i.e., fear of death and disease, bodily focus, worry and concern about illness, frequency of treatment, decisions to seek treatment, and physical illness symptoms). These researchers hypothesized that greater sense of humor may contribute to more positive perceptions of physical health. Data collected from a sample of undergraduates indicated higher humor is not correlated with any of the health behaviors measured in this study. However, 
humor did positively correlate with perceptions of health, i.e., a higher level of humor corresponded to a higher level of perceived health. These findings help explain why the popularity of humor as a powerful medicine despite mixed results from empirical research.

As with several of the other categories of literature concerning humor and physical health, the results of research concerning humor and self-reported physical illness symptoms have been mixed. Collectively, these results suggest the connection between humor and physical illness symptoms may be moderated by other variables. In his review of the literature concerning a link between physical health and humor, Martin (2001) describes some methodological concerns that will be discussed later in the section.

\section{Humor, Laughter, and Longevity}

To date very little empirical research has been conducted to explore any possible connection between humor and longevity. If humor does confer health benefits, then individuals who use more humor should live longer lives, ceteris paribus. The little research conducted so far does not support this hypothesis. In fact, there is some evidence that people who use more humor actually die sooner than those who use less humor.

So far only two empirical studies have been conducted. Assuming humor might make people who use it live longer, the age of death for famous comedians, comedy writers, and humorous authors has been compared with the age of death for serious entertainers and authors. There was no significant difference between these two groups (Rotton, 1992). Similar results were obtained when Friedmen et al. (1993) examined data collected from the Terman Life Cycle Study. Parents and teachers gave participants at 
the age of 12 ratings of humor and optimism, which were combined into a measure of cheerfulness. Surprisingly, those with higher levels of cheerfulness also had higher levels of mortality at each stage in life. These results suggest humor does not help a person live longer, as has been hypothesized previously.

This research was conducted with the assumption that all humor is adaptive. Other research measuring both adaptive and maladaptive styles of humor has found a correlation between humor style and self-report measures of health. Using the Humor Styles Questionnaire, Martin et al. (2003) correlated humor styles with scores on a physical symptom checklist. These researchers found maladaptive humor styles positively correlated with scores on the physical symptom checklist, while adaptive humor styles were negatively correlated with scores on the physical symptom checklist. Martin et al. (2003) points out the correlations found in this study were much stronger than those found in previous research thus accounting for more variability. These results were replicated using a sample of Chinese university students reported by Chen and Martin (2007). The results from these two studies suggest there may indeed be a relationship between humor and health when humor is divided into adaptive and maladaptive categories.

Martin $(2001,2002,2004)$ conducted an exhaustive review of available research (including many of the studies mentioned above) examining the connection between humor and physical health. While claims stating that having a sense of humor can improve health have existed for several millennia, empirical research has shown mixed results. Taking into consideration several methodological concerns Martin (2001) concluded there is not enough evidence to support the claim that humor enhances 
physical health. With the development of a newer humor scale, which distinguishes between adaptive and maladaptive styles of humor, Martin (2004) and Martin et al. (2003) suggest there may indeed be a connection between humor and health which has been elusive to many previous researchers

One explanation for the lack of consistent evidence supporting a connection between humor and physical health are some methodological issues raised by Martin (2001). These methodological concerns varied depending on the topic of research. For example, he expressed concern in the studies examining the connection between humor and immunity because of issues like small sample sizes, variability in immune marker measured, and use of different statistical tests to analyze data collected (correlations, ANOVAs, and non-parametric tests). With regard to humor and self-reported illness symptoms, Watson and Pennebaker (1989) advise against self-report illness symptom checklists because results are often influenced by the participants' neuroticism. According to Martin (2001) correlating the results of symptom checklists and humor is less desirable in health research because neuroticism confounds the results. Sense of humor typically correlates negatively with neuroticism (Kohler \& Ruch, 1996). Martin (2001) suggests any negative correlation between humor and physical symptoms may be due to a shared neuroticism component instead of any health benefit of engaging in humor.

A second explanation for this lack of consistent empirical evidence validating a positive correlation between humor and health could have to do with the self-report humor measures used. The self-report humor measures used in most studies examining the link between humor and health did not distinguish between adaptive and maladaptive 
uses of humor (Kuiper \& Martin, 1998; Martin, 2001). Previous self-report measures of humor contained items presumably endorsed only by those with adaptive styles of humor; however, it is also possible for those with maladaptive styles of humor to endorse these items as well. The Humor Styles Questionnaire (HSQ; Martin et al., 2003) explicitly measures both adaptive and maladaptive types of humor. If both adaptive and maladaptive types of humor can measured accurately, it may be possible to identify a stronger link between humor and physical health. New evidence, provided by Martin et al. (2003) and Martin and Chen (2007) suggests this connection may indeed exist; however, more research is needed to explore this possible connection further using better methodology.

\section{Humor and Mental Health}

While a humor-physical health connection has not been consistently shown in previous research, humor has been linked to indicators of mental health and well-being. Humor has been shown to help make people feel better emotionally. Even before there was a measure to differentiate between adaptive and maladaptive styles of humor, research showed self-report humor scales correlated significantly with several indicators of psychological health (i.e., depression ratings, self-esteem, psychological well-being; for a comprehensive review of this research see Martin, 1996). This section will only focus on studies involving the Humor Styles Questionnaire (Martin et al., 2003). These studies can be divided into three categories: those that related to social adjustment, those that related to personality and those that related to psychological adjustment. Previous research regarding mental health and humor styles will be outlined in the respective categories below. 


\section{Social Adjustment}

During the process of constructing the HSQ, Martin et al. (2003) hypothesized the adaptive forms of humor, especially Affiliative humor, would correlate positively with measures of healthy social adjustment while the maladaptive forms of humor would correlated positively with measures of unhealthy social adjustment. Since then, researchers have investigated the relationship between humor styles and social adjustment by looking for correlations with constructs like attachment style, conflict style, emotional intelligence, social competence, relationship satisfaction, and shyness. The thread that ties these constructs together is how they influence interpersonal interactions.

One of the first researchers to examine the relationship between humor styles and social adjustment was William Hampes. In the course of two studies he explored the relationship between humor styles, shyness, and loneliness. In his first study, Hampes (2006) investigated the relationship between shyness and humor. This researcher determined there is a negative correlation between shyness and affiliative humor, and a positive correlation between shyness and self-defeating humor. These relationships can be explained by the likelihood of shy people to use affiliative humor when unable to relax in social situations. In a follow-up study, Hampes (2005, Note: these two studies were published in different journals, and the follow up study was actually published first) investigated the role of loneliness in the correlation between shyness and humor style. This follow-up study found loneliness to be significantly and positively correlated with 
shyness. Additionally, both adaptive humor styles correlated negatively with loneliness while self-defeating humor correlated positively. These two studies establish there is a relationship between shyness, loneliness, and humor styles. They suggest that shy people may not know how to use adaptive styles of humor and instead endorse maladaptive styles of humor. Higher use of the maladaptive styles of humor might alienate the shy person from others thereby increasing his or her levels of loneliness.

Both of these studies were correlational in nature so it is impossible to determine causality; another group of researchers continued the investigation into the connection between shyness, loneliness, and humor styles. Using another sample of university students Fitts, Sebby, and Zlokovich (2009) replicated and extended Hampes’ (2005, 2006) results in a subsequent study by constructing regression models. The regression model that yielded the best predictive value helped these researchers establish a sequence beginning with shyness, progressing to humor style, and ending with loneliness. The data suggest an individual's shyness leads to his or her higher endorsement of maladaptive styles of humor, which pushes people away and leads to increased levels of loneliness. Taken together, this line of research suggests humor is important in the ability to make and maintain relationships with other people, and the use of maladaptive styles of humor may make developing meaningful relationships difficult.

Using a sample of university students, Yip and Martin (2006) sought to determine potential relationships between humor styles, emotional intelligence, and social competence. As expected, these researchers found emotion management (a component of emotional intelligence) correlated positively with self-enhancing humor. Social competence was positively correlated with both adaptive styles of humor. Additionally, 
maladaptive humor styles correlated negatively with social competence. The researchers concluded adaptive styles of humor facilitate social interaction indirectly because their presence suggests the lack of maladaptive humor styles. Based on the data from this study, the presence of maladaptive humor styles seems to make social interaction difficult.

Expanding on previous literature, researchers have also examined possible relationships between attachment styles (secure, avoidant, disorganized, and anxious), conflict styles (dominating, obliging, avoiding, and integrating), humor styles (affiliative, self-defeating, aggressive, and self-enhancing), and relationship satisfaction (Cann, Norman, Wellbourne, \& Calhoun, 2008). These researchers found the insecure styles of attachment correlated positively with the maladaptive styles of humor while correlating negatively with the adaptive styles of humor. Secure attachment was positively correlated with the adaptive styles of humor and negatively correlated with the maladaptive styles. They also found the healthy conflict style correlated positively with the adaptive styles of humor, while the less healthy conflict styles correlated positively with the maladaptive styles of humor. After considering the results of this study, Cann et al. (2008) did not correlate relationship satisfaction directly with humor styles and concluded that together humor styles and conflict styles help mediate the relationship between attachment style and relationship satisfaction. These findings suggest those with healthy conceptions of self and others tend to endorse adaptive styles of humor. These findings are in line with the results of previous studies and add weight to the notion that the use of the adaptive humor styles may facilitate the development and maintenance of meaningful relationships with others. 
The research reviewed here has hypothesized that humor styles play a role in fostering new relationships and maintaining current relationships. Previous research has included individuals rather than romantically involved couples. Taking a different approach to studying how humor styles impact social adjustment, Hall (2010) examined heterosexual couples in an attempt to determine how humor style may contribute to embarrassment experienced by a romantic partner. Using a survey and an experimental design, Hall found aggressive humor is positively correlated with embarrassment. Consistent with Martin et al. (2003), this study found men tend to use aggressive humor more than women. As expected, Hall (2010) found that women tend to feel more embarrassed than men in response to aggressive humor. In addition, self-defeating humor is more likely to be used by the embarrassed partner, but self-enhancing humor is associated with less embarrassment. These findings suggest adaptive humor styles are positively correlated with satisfaction in romantic relationships.

Several studies have found evidence to suggest that humor styles and relationship satisfaction are correlated. Following up on the results reported by Hall (2010) another group of researchers conducted a study to explore whether there is a direct relationship between humor styles and relationship satisfaction. Cann, Zapata, and Davis (2011) asked a sample of heterosexual couples to participate in a study. Each participant completed an HSQ and a relationship satisfaction measure. In addition to these measures, participants were asked to complete an HSQ from the point of view as his or her partner. While there was very little similarity found between partner's self-reported humor styles, Cann et al. (2011) found relationship satisfaction was best predicted by perceptions of a partner's humor style, rather than the partner's actual self-reported 
humor style or the participant's own humor style. Additionally, perception of an affiliative or self-enhancing humor style predicted the highest relationship satisfaction while perception of an aggressive humor style predicted the lowest relationship satisfaction.

These studies all seem to support the hypotheses of Martin et al. (2003). A common finding in the previously described research is the positive correlation of the adaptive humor styles with healthier forms of social interaction, as well as a positive correlation of the maladaptive humor styles with less healthy forms of social interaction. The data from these studies collectively suggest those who endorse adaptive styles of humor tend to have healthier forms of interacting with others and are more likely to develop relationships with other people and view their relationships as satisfying and meaningful. In addition to indicators of social adjustment, humor styles have also been shown to correlate with personality traits, which have an impact on both psychological and social adjustment.

\section{Personality}

While developing the HSQ, Martin et al. (2003) hypothesized the different humor styles would each correlate with the Big 5 personality traits. After constructing the HSQ, Martin and his colleagues administered the HSQ and Revised NEO Personality Inventory to a sample of university students. These researchers found Openness and Extraversion to be positively correlated with both adaptive styles of humor, Agreeableness and Conscientiousness to be negatively correlated with both maladaptive styles of humor, and Neuroticism to be negatively correlated with self-enhancing humor and positively 
correlated with self-defeating humor. The findings support the hypotheses set forth by these researchers.

Using a behavioral genetic approach with a sample of monozygotic twins, Vernon, Martin, Schermer, and Mackie (2008) investigated humor styles and the Big 5 personality traits (Openness, Conscientiousness, Extroversion, Agreeableness, and Neuroticism). These researchers found the humor styles are attributable to genetic and non-shared environment factors. When examining the relationships between the Big 5 and humor styles they found results similar to those of Martin et al (2003). These results have been replicated by using a sample consisting of college students and professional comedians (Greengross, Martin, \& Miller, 2011).

Taken together, the results from these three studies examining the correlation between humor styles and the Big 5 personality traits offer insights into the ways differing levels of these personality traits impact the way individuals employ humor styles. Vernon et al. (2008) concluded the various relationships between personality traits and the humor styles may explain the relationships between the humor styles and various indicators of psychological adjustment. The Big 5 are not the only personality traits that have been correlated with the humor styles. Three other groups of researchers have explored the connection between humor styles and some so-called aversive personality traits (e.g., narcissism).

The first group of researchers to study the relationship between humor styles and aversive personality traits was interested in the relationship between humor styles and the Dark Triad (narcissism, psychopathy, and Machiavellianism; outlined by Paulhus \& Williams (2002)). Veselka, Schermer, Martin, and Vernon (2010b) measured these traits 
in a small group of monozygotic and dizygotic twins. These researchers found psychopathy and Machiavelianism are positively correlated with the aggressive and selfdefeating humor styles. Narcissism had a weak, positive correlation with affiliative humor. These researchers concluded that narcissism could help to establish and maintain social bonds to a degree.

Another group of researchers was inspired by the results of Veselka et al. (2010b) and sought to replicate and extend the study. Martin, Lastuk, Jeffery, Vernon, and Veselka (2012) examined not only the correlations between humor styles and the global traits that make up the dark triad but also considered the sub-factors that make up the dark triad (e.g., callous affect which is a sub-factor of psychopathy). The correlations between humor styles and the global traits of narcissism, psychopathy, and Machiavellianism were very similar to those found by Veselka et al. (2010b). As expected, Martin et al. (2012) also found several correlations between several sub-factors within the dark triad and humor styles (e.g., positive correlation between callousness and aggressive humor).

Similar to Veselka et al. (2010b), Zeigler-Hill and Besser (2011) investigated whether humor styles mediate the association between pathological narcissism and selfesteem. These researchers differentiated between grandiose narcissism and vulnerable narcissism based on Pincus and Lukowitsky (2010). Grandiose narcissism can be easily recognized by a tendency of an individual towards self-aggrandizement, exploitation of others, and exhibition consistent with Narcissistic Personality Disorder diagnostic criteria set forth by the Diagnostic and Statistical Manual IV (DSM IV; American Psychiatric Association, 2000; Pincus \& Lukowitsky, 2010). On the other hand, Pincus and 
Lukowitsky (2010) define vulnerable narcissism as poor self-regulation, which leads to self-criticism, negative affective experiences, and interpersonal problems. Zeigler-Hill and Besser (2011) found grandiose narcissism was positively correlated with the affiliative humor style while vulnerable narcissism was positively correlated with maladaptive the humor styles. These researchers concluded people who score higher in grandiose narcissism score higher on affiliative humor probably in an effort to increase their social standing. Finally, these researchers concluded humor styles appear to mediate the association between narcissism and self-esteem.

These investigations into the relationship between personality traits and humor styles largely supported the predictions of Martin et al. (2003) with a few unexpected twists. The common thread that binds these results is the finding that adaptive styles of humor correlate positively with personality traits that predict levels of social and psychological adjustment. A final category of humor and mental health research remaining to be reviewed is the relationship between humor styles and indicators of psychological adjustment (e.g., psychological distress, depression, and self-esteem).

\section{Psychological Adjustment}

In the process of validating the HSQ, Martin et al. (2003) also administered several measures meant to assess mood, well-being, and social relationships, and found that adaptive humor styles were significantly and positively correlated with constructs like positive self-esteem, social intimacy, and well-being. They also found that maladaptive styles of humor were positively and significantly correlated with constructs like aggression, hostility, and physical/psychological symptoms. These findings have been replicated in several follow-up studies. 
Depression was one of the first indicators of psychological adjustment to be investigated regarding to a possible relationship with humor styles. Several groups of researchers have approached the study of this relationship from a few different perspectives. The first group of researchers investigated the role humor plays in mediating the relationship between attribution style (i.e., the way people tend to explain life events) and dysphoria. These researchers (Hugelshofer, Kwon, Reff, \& Olsen, 2006) found results consistent with previous findings: adaptive styles of humor were negatively correlated with depressive symptoms, while the maladaptive styles of humor were positively correlated with depressive symptoms.

Two studies followed Hugelshofer et al. (2006) regarding a connection between humor style and depression. One study approached the relationship between depression and humor styles from a personality-vulnerability perspective while the other was interested in early maladaptive schemas. Investigating the relationship between personality vulnerability to depression and humor styles, Frewen et al., (2008) found depressive symptoms were negatively correlated with both the affiliative and selfenhancing humor styles and positively correlated with self-defeating humor. The other study conducted by Dozois, Martin, and Bieling (2009), found that adaptive humor styles were negatively correlated with each of the domains measured by the early maladaptive schemas measure. The maladaptive styles of humor positively correlated with all the early maladaptive schema domains. These researchers concluded the relationship between most early maladaptive schema domains and depressed mood was mediated by both self-enhancing and self-defeating humor styles. Taken together, the results from 
each of these studies suggest that adaptive styles of humor might help to reduce symptoms of depression while maladaptive styles of humor might increase them.

Depression is only one of the indicators of psychological adjustment correlated with humor styles (Martin et al., 2003). While investigating the role of humor in emotional burnout, Tümkaya (2007) studied a sample of university lecturers to determine if there is a correlation between humor style and level of burnout. The results of this study showed there is a positive correlation between both aggressive humor and selfdefeating humor and emotional burnout. Additionally, affiliative and self-enhancing humor styles were negatively correlated with emotional burnout. These results were replicated with using a sample of active physicians. Wojtyna and Stawiarska (2009) found difficult psychosocial working conditions enhance burnout symptoms. They also found adaptive humor styles correlate negatively with burnout symptoms, while the maladaptive humor styles correlate positively with burnout symptoms. The findings from these studies collectively suggest that having an adaptive humor styles might help to protect one from developing emotional burnout.

The results from studies mentioned previously increased interest in the area of psychological adjustment and humor styles. Researchers began to examine the factors that underlie depression and emotional burnout. Adaptive humor styles have been shown to be positively correlated with positive self-evaluative standards and psychological wellbeing, while maladaptive styles of humor have been positively correlated with negative self-evaluative standards and psychological distress (Kuiper \& McHale, 2009). Another group of researchers replicated these results with a sample of university students from Hong Kong. Yue et al., (2010) also found that affiliative and self-enhancing styles of 
humor were significantly and positively correlated with optimism and negatively correlated with psychological distress. On the other hand, aggressive and self-defeating styles of humor were significantly and positively correlated with psychological distress. Findings like this suggest humor can be adaptive and maladaptive in other cultural settings.

Humor style and resiliency have also been linked. Using a sample of twin pairs in a behavioral genetic design, Veselka, Schermer, Martin, and Vernon (2010a) found adaptive styles of humor positively correlated with all mental toughness factors while the maladaptive humor styles correlated negatively with all mental toughness factors. Shortly after this study was published another group of researchers, Stieger et al. (2011) investigated the relationship between humor styles, implicit self-esteem, and explicit selfesteem. These researchers found adaptive humor styles are positively correlated with explicit and implicit self-esteem. There was no significant correlation between the aggressive humor style and either explicit or implicit self-esteem; however, self-defeating humor correlated negatively with both explicit and explicit self-esteem. Said another way, individuals who use adaptive styles of humor tend to be better at coping with adversity and tend to have better views of self. Results like this add even more weight to the notion that healthy psychological adjustment is associated with the use of adaptive humor.

Considered together, there is an abundance of support for the notion put forth by Martin et al. (2003) that humor can be both adaptive and maladaptive. The research literature consistently demonstrates that adaptive humor is positively correlated with indicators of higher levels of mental health (e.g., higher levels of social competence, 
higher self-esteem, and lower levels of neuroticism). At the same time, maladaptive humor has been consistently linked to indicators of lower levels of mental health (e.g., more depressive symptoms, higher levels of shyness and loneliness, and lower levels of resiliency). Moreover, these results give some insight into how endorsement of humor style (whether adaptive or maladaptive) can influence an individual's mental health and quality of life.

\section{Quality of Life}

Like humor, health related quality of life is not easily defined, nor a simple, single domain (Ferrans, 1996). Throughout the history of the concept of quality of life there have been multiple perspectives with regard to how it should be measured. From an economic perspective, quality of life is determined by one's standard of living (Asthana, 2009). Based on this perspective, quality of life can be increased if standard of living goes up. Along these lines, income and wealth yield satisfaction and happiness (Asthana), though only to a certain point (Diener \& Biswas-Diener, 2002). In a metaanalysis of 916 surveys, Myers (2000) found that financial status has a limited effect on levels of happiness.

Starting in the 1980s, quality-of-life researchers began to consider factors outside of standard of living (WHO, 1991). Researchers began to examine the impact of poor health indicators (e.g., disease) on behavior and daily activities (WHO). While accounting for more influences, these measures did not fully assess quality of life because they assessed how negative health factors decrease quality of life (Fallowfield, 1990). In response, the World Health Organization Quality of Life (WHOQoL; 1998) work group developed a scale meant to assess quality of life with a more holistic approach. This 
health and health care approach to quality of life measurement is called health related quality of life (WHOQoL, 1998).

For the purposes of this thesis, the definition of quality of life put forth by the World Health Organization (WHO) shall be used. WHOQoL (1998) work group defines quality of life as "individuals' perceptions of their position in life in the context of the culture and value systems in which they live in and in relation to their goals, expectations, standards and concerns" (p. 299). Quality of life is influenced by physical health, level of independence, social relationships, and salient features in the environment (Skevington et al., 2004).

Currently, there are several different instruments designed to measure healthrelated quality of life and its multiple domains, for example, the Comprehensive Quality of Life Scale developed by Cummins, McCabe, Romeo, and Gullone (ComQol, 1994). Health-related quality-of-life instruments can be designed for a specific population (e.g., college students), or for a specific condition (e.g., cognitive impairment). According to Higginson and Carr (2001), health-related quality-of-life measures have several valid uses in clinical settings. According to these researchers, they can be used to prioritize problems, facilitate communication, screen for potential problems, identify patient preferences, and monitor changes or responses to treatment.

To date, no published studies have directly examined the relationship between humor styles and quality of life. The research reviewed previously seems to indicate there is a relationship between the adaptive humor styles and indicators of mental health. Since mental health is a component of quality of life, it can be inferred that an individual's humor style may be related to his or her quality of life. 


\section{Quality of Life and Humor Styles}

Currently, there is not much in the research literature about a connection between humor and quality of life. The research that has been published has only hinted at the possibility that humor and quality of life are connected. For example, Thorson and Powell (1993b) measure personality and humor. These researchers found there was no significant difference in humor creation among all age groups measured (ages 18-90), and significant correlations were found between factors of the humor scale and some personality traits. For example, higher humor was significantly and negatively correlated with traits like deference and order, while significantly and positively correlated with traits like exhibition and dominance (Thorson \& Powell). Based on these results the researchers concluded that humor, as a multidimensional concept, seems to be intimately related to quality of life. These results were replicated in another study (Thorson, Powell, Sarmony-Schuller, \& Hampes, 1997).

Kuiper, Martin, and Dance (1992) attempted to determine if sense of humor could enhance quality of life measured through life satisfaction. To accomplish this, the researchers assessed sense of humor, personal role evaluations, positive affect, and positive/negative life events from the previous two weeks. They found those participants who rated their personal roles as more pleasant and satisfying had higher humor scores. In addition, higher affect was positively and significantly correlated with sense of humor even when these participants were confronted with negative life events. Thus the 
researchers concluded that sense of humor facilitates a more positive orientation toward life in general and therefore a higher quality of life (Kuiper et al., 1992).

Since health-related quality-of-life takes into account one's physical and mental health, social relationships, and salient environmental factors, the research reviewed in the sections regarding humor and health (both physical and mental) support the suggestion that humor will correlate with quality of life. Quality-of-life scales ask about

one's subjective view of his or her health. Results obtained by Kuiper and Nicholl (2004) suggest more use of adaptive humor will predict a higher subjective physical health rating. All the literature reviewed regarding humor styles and psychological adjustment suggests endorsement of adaptive humor will positively correlate with psychological quality of life. On a similar note, the results in the literature regarding humor styles and social adjustment suggest adaptive humor styles will correlate positively with the social domain in quality of life.

\section{Conclusion}

Humor has been shown to be a complex and multifaceted concept. Throughout history theorists have developed several different ideas and theories in an effort to define humor and why it is used. Based on these theories, several conceptualizations of humor have arisen. Humor has long been regarded as the best medicine for both ailments of the body and the mind. Anecdotal evidence provided by Cousins (1979) helped to jumpstart interest in empirical research to establish a connection between humor and health.

Until recently, humor was assumed to be used in only an adaptive way, so researchers assumed individuals who use more humor would show signs of increased physical health (e.g., increased pain tolerance). However, research has not been able to 
find a consistent connection between humor and physical health. Martin et al. (2003) suggests this last of a connection may be due at least in part to studies that did not differentiate between adaptive and maladaptive forms of humor. In response to a lack of a humor assessment designed to distinguish between adaptive and maladaptive humor, Martin et al. (2003) conducted an exhaustive review of the literature to find evidence of the adaptive and maladaptive forms of humor and built an assessment to distinguish the two called the HSQ.

While the connection between humor and physical health has been elusive, there is a positive and significant correlation between the use of humor and indicators of psychological wellbeing (e.g., lower levels of stress). This connection was established even before the HSQ was developed. Research using the HSQ has supported the notion that humor can be adaptive or maladaptive. Subsequently, adaptive humor has been consistently and positively correlated with indicators of mental health (e.g., higher levels of resiliency) while maladaptive humor has been consistently and negatively correlated with indicators of mental health (e.g., relationship satisfaction). Based on this previous research, there is enough evidence to hypothesize a connection between humor and quality of life.

Quality of life, like humor, is also a multifaceted concept. While quality of life used to be measured only by standard of living, it has evolved to encompass several aspects of healthy living including: physical health, psychological health, social relationships, and salient features of the environment. To date there are no studies directly supporting the relationship between humor and quality of life; however, several 
studies have suggested there is a positive correlation between adaptive humor and quality of life and several of its domains.

\section{Research Questions}

To date, most studies have focused on correlations between humor styles and indicators of health (Kuiper \& McHale, 2009; Stieger et al., 2011; Yue et al., 2010). Establishing a regression model with high predictability is the next step in exploring the relationship between humor styles and quality of life. If quality of life can be predicted by adaptive styles of humor, then it may be possible to develop a treatment approach aimed at altering one's humor style to improve his or her quality of life.

\section{Hypotheses}

After considering previous research in the areas of humor styles and quality of life and keeping in mind the research questions guiding this study, two hypotheses emerge.

1. The first is that adaptive humor styles (Affiliative and Self-enhancing) will predict higher quality of life, while maladaptive humor styles (Aggressive and Self-defeating) will predict lower quality of life.

2. The second is that adaptive humor styles will predict higher domain scores (physical, psychological, social, and environment) within quality of life, while maladaptive humor styles will predict lower domain scores within quality of life. 
"Analyzing humor is like dissecting a frog. Few people are interested and the frog dies of

it."

--E. B. White

\section{CHAPTER THREE}

\section{Methods}

\section{Participants}

Participants were undergraduate students currently attending a mid-size university. Participants were sought through the psychology department's research participant pool. Participation was voluntary and was compensated with course credit (participating in a research study was one option to obtain course credit, however there were other opportunities to get the same course credit).

This sample was comprised 100 individuals. Participants in this study ranged in age from 18 to 26 years while the average age of participants was 18.48 years $(S D=$ $.973)$. There were 44 (44\%) males, 55 (55\%) females, and $1(1 \%)$ person identified as other. Most of the participants $(n=62)$ in this study identified as Caucasian or White, 19 identified as Asian-American (19\%), 11 identified as Biracial (11\%), and 8 identified as Latino/a ( $8 \%)$. The vast majority $(n=90,90 \%)$ of this sample were college freshman in terms of class status, with 5 sophomores (5\%), 2 juniors ( $2 \%)$, and 3 seniors (3\%). Finally, 65 (65\%) of the participants reported personal and/or parental income of over $\$ 75,000 /$ year or more while 35 (35\%) of the participants reported personal and/or parental income of $\$ 75,000 /$ year or less. 


\section{Materials}

\section{Humor Styles}

The Humor Styles Questionnaire (Martin et al., 2003) was used to measure adaptive and maladaptive styles of humor. The 32-item measure consists of 4 subscales; 2 are considered adaptive and two are considered maladaptive. The adaptive subscales include items to measure Affiliative humor (e.g., "I don't have to work very hard at making other people laugh-I seem to be a naturally humorous person.”) and Selfenhancing humor (e.g., "I don't need to be with other people to feel amused - I can usually find things to laugh about even when I'm by myself.”). The maladaptive subscales include items designed to measure Aggressive humor (e.g., "When telling jokes or saying funny things, I am usually not very concerned about how other people are taking it.") and Self-defeating humor (e.g., "I let people laugh at me more make fun at my expense more than I should.”). Martin et al. (2003) showed strong psychometrics for this scale with internal reliability coefficients for all four subscales ( $\alpha$ ranging from .77 to .81 ). Responses are made on a seven-point Likert scale where $1=$ Totally Disagree and 7 $=$ Totally Agree.

\section{Quality of Life}

The World Health Organization Quality of Life-Brief version (WHOQOL-BREF; (WHOQoL Group, 1998) is a 26-item measure that was created to serve as a shorter version of the World Health Organization Quality of Life 100. The WHOQOL-BREF measures subjective quality of life in four domains: Domain 1/Physical (e.g., To what extent do you feel that physical pain prevents you from doing what you need to do?), Domain 2/Psychological (e.g., How much do you enjoy life?), Domain 3/Social (e.g. 
How satisfied are you with your personal relationships?), and Domain 4/Environmental (e.g., How safe do you feel in your daily life?). In addition to these domains, the WHOQOL-BREF asks one question related to overall quality of life and one question related to overall quality of health. Internal consistency for Domains 1,2 , and 4 has been shown to be acceptable (.8 to .82 ) while Domain 3 was around .68 (Skevington et al., 2004). Responses were made on a 5-point Likert scale with lower scores indicating lower quality of life and high scores indicating a high quality of life.

\section{Demographics}

Participants were asked to provide demographic information including their age, gender, ethnicity and race, personal and/or parental income, and class standing.

\section{Procedure}

Data were collected through an online survey. Participants who were interested in participating in the study signed up through Cal Poly's online study website. Shortly after receiving confirmation of participation in the study, a link was sent via email to the student. To participate in the study students either clicked the link or copied it into the Url window in their web browser. Before starting the survey participants saw the informed consent form explaining the purpose of the study. Participants were also informed of their right to withdraw from the study at any time. To continue with the study, participants were required to indicate they were choosing to participate of their own freewill. If someone indicated he or she did not wish to participate, the survey was canceled and he or she was thanked for their consideration to participate in the study. Filling out the questionnaire form took an average of 20 to 30 minutes. Upon completing 
the questionnaire, participants were given a written debriefing and provided with a prompt for a writing assignment required to receive credit in class.

\section{Statistical Analyses}

To determine which of the four humor styles significantly contributed to the prediction of quality of life, a stepwise regression was conducted with the World Health Organization Quality of Life Brief version score as the dependent measure. Each humor style was entered as an independent variable. After determining how the humor styles predict overall quality of life other stepwise regression analyses were performed to determine which predicted each domain (physical, psychological, social, and environment) within the quality-of-life scale. 
"Common sense and a sense of humor are the same thing, moving at different speeds. A sense of humor is just common sense, dancing."

--William James

\title{
CHAPTER FOUR
}

\begin{abstract}
Results
Before testing the hypotheses of this study, the internal reliability of the two scales, Humor Styles Questionnaire (HSQ: Martin et al., 2003) and World Health Organization Quality of Life Brief (WHOQOL-Bref: WHOQoL Group, 1998), was assessed. For this sample, the internal reliability coefficients for each of the four humor styles were similar to those found by Martin et al. (2003). Cronbach's alphas for the Affiliative, Self-Enhancing, Aggressive, and Self-Defeating subscales were .70, .80, .70, and .79 respectively. For the WHOQOL-Bref, Cronbach's alpha coefficients for the various domains were similar to those found by Skevington et al., (2004), with coefficients of $.70, .79, .62$, and .71 for the physical, psychological, social, and environmental domains respectively. The internal reliability coefficients were determined to be sufficient (George \& Mallery, 2003) to proceed with further statistical analysis.

A series of five multiple regression models with stepwise entry was conducted to find the best model to predict quality of life and its domains. Each of the four humor styles (affiliative, self-enhancing, aggressive, and self-defeating) was entered as a predictor variable, while the quality of life scores (overall quality of life, physical, psychological, social, and environmental) were entered as dependent variables. In order to reduce the chance of making a Type I error, alpha was reduced from .05 to .01 . SPSS version 19.0 was used to analyze the data collected in this study.
\end{abstract}




\section{$\mathrm{H}_{1}$ : Endorsement of adaptive styles of humor will positively predict overall quality}

of life while endorsement maladaptive styles of humor will negatively predict overall quality of life.

To determine which of the four humor styles predict quality of life a multiple regression using a stepwise method was utilized. The final model included selfenhancing humor and self-defeating humor. Self-enhancing humor positively predicted overall quality of life and self-defeating humor negatively predicted quality of life, $(F(2$, $97)=13.30, p<.01$. Combined, these humor styles accounted for $21.5 \%$ of the variance. The other humor styles (affiliative and aggressive) both predicted quality of life in the predicted direction; however, these results were not significant.

\section{$\mathrm{H}_{2}$ : Endorsement of the adaptive styles of humor will positively predict each of the domains of quality of life while endorsement of maladaptive styles of humor will negatively predict these domains.}

In order to determine how the four humor styles predict each of the quality of life domains, four additional multiple regressions using a stepwise method were conducted. Self-enhancing humor contributed significantly and positively to the physical domain $(F(1,98)=12.58, p<.01)$ and accounted for $11.4 \%$ of the variance. Two humor styles significantly predicted the psychological domain; self-enhancing was positively related and self-defeating humor was negatively related to the psychological health domain $(F(2$, $97)=16.44, p<.01)$. Together, self-enhancing and self-defeating humor accounted for $25.3 \%$ of the variance in the psychological domain. Two humor styles significantly predicted the social domain, affiliative humor was positively related while aggressive humor was negatively related this domain $(F(2,97)=8.46, p<.01)$. Collectively, 
aggressive and affiliative humor accounted for $14.9 \%$ of the variance in the social domain. Self-enhancing humor significantly and positively predicted the environmental domain $(F(1,98)=8.31, p<.01)$, accounting for $7.8 \%$ of the variance.

While not all of the humor styles significantly predicted the domains within quality of life, they did predict domain scores in the hypothesized direction. However, there was one exception; in the physical domain aggressive humor positively, but very weakly, predicted the domain score. This finding, while unexpected, was not significant. Please see Table 1 for more details about these regression models.

Table 1: Summary of regression analyses

\begin{tabular}{lcc}
\hline & $\boldsymbol{\beta}$ & $\mathbf{t}$ \\
\hline Quality of Life & & \\
Affiliative & .176 & 1.810 \\
Self-enhancing & $.417^{* *}$ & 4.613 \\
Aggressive & -.128 & -1.335 \\
Self-defeating & $-.247^{* *}$ & -2.731 \\
Physical & & \\
Affiliative & .079 & .763 \\
Self-enhancing & $.337^{* *}$ & 3.546 \\
Aggressive & .051 & .531 \\
Self-enhancing & -.119 & -1.252 \\
Psychological & & \\
Affiliative & .160 & 1.682 \\
Self-enhancing & $.406^{* *}$ & 4.610 \\
Aggressive & .068 & -.728 \\
Self-defeating & $-.337^{* *}$ & -3.829 \\
Social & & \\
Affiliative & $.272^{* *}$ & 2.904 \\
Self-enhancing & .136 & 1.333 \\
Aggressive & $-.274^{* *}$ & -2.920 \\
Self-defeating & -.104 & -1.038 \\
Environment & & \\
Affiliative & .113 & 1.066 \\
Self-enhancing & $.280^{* *}$ & -1.982 \\
Aggressive & -.185 & -1.505 \\
Self-defeating & -.146 & \\
\hline A $p<.05, * *$ & & \\
\hline
\end{tabular}


"If I had no sense of humor, I would long ago have committed suicide."

--Mahatma Gandhi

\section{CHAPTER FIVE}

\section{Discussion}

Two hypotheses emerged from the literature currently available concerning humor styles (affiliative, self-enhancing, aggressive, and self-defeating) and quality of life. The first was that adaptive styles of humor would positively predict overall quality of life while the maladaptive styles of humor would negatively predict overall quality of life. Going a step further, the second hypothesis was that adaptive humor styles would positively predict each of the domains (physical, psychological, social, and environmental) within quality of life while the maladaptive humor styles would negatively predict these domains. Both hypotheses were partially supported in this study. Effect sizes found in this study ranged from moderate to large based on guidelines put forth by Cohen (1988).

It was originally predicted that both adaptive styles of humor would positively and significantly predict quality of life and its domains. However, in each model only self-enhancing humor (with the exception of the social domain) predicted quality of life and its domains. Affiliative humor did relate quality of life and its domains in the predicted direction, but the results were not significant (again, with the exception of the social domain). Additionally, the hypotheses predicted the maladaptive styles of humor would predict quality of life and its domains in a negative direction, however, this was not the case. Instead, self-defeating humor negatively predicted overall quality of life and the psychological domain, while aggressive humor negatively predicted the social 
domain. On the remaining variables the maladaptive styles of humor predicted quality of life and its domains in the expected direction; however, the results were not significant.

Taken together, these results provide evidence that quality of life can be partially predicted based on humor style scores. The humor styles that significantly predict quality of life and most of its domains can be described as self-oriented, i.e., self-enhancing and self-defeating. The findings of this study are in line with some previous research. The connection between humor and physical health has been inconsistent (Martin, 2001), with some studies supporting the notion that humor has health benefits (e.g., Dillon et al., 1985; Hudak et al., 1991; Lefcourt et al., 1997) and other that don’t (e.g., McClelland \& Cheriff, 1997; Nevo et al., 1993; White \& Camarena, 1989). This study found that adaptive humor does indeed positively predict subjective perceptions of physical health. Humor has been shown to be consistently associated with indicators of mental health. Adaptive humor has been consistently associated with higher levels of relationship satisfaction (Cann et al., 2011), self-esteem (Stieger et al., 2011), and resiliency (Veselka et al., 2010a). On the other hand, maladaptive humor has been consistently associated with higher levels of depression (Dozois et al., 2009; Frewen et al., 2008; Hugelshofer et al., 2006), narcissism (Martin et al., 2012; Veselka et al., 2010b; Zeigler-Hill \& Besser, 2011), and shyness (Hampes, 2005; 2006). These results have been replicated and extended in this study.

Much of the previous research exploring the connection between humor styles and indicators of mental health used correlational designs (e.g., Kuiper \& McHale, 2009; Stieger et al., 2011; Yue et al., 2010). This study sought to expand on previous research by examining how humor styles can predict a more global indicator of well-being, i.e., 
quality of life. In addition to examining how humor styles predict quality of life, this study sought to explore how humor styles predict the domains that make up quality of life. By examining how humor styles predict the domains of quality of life, this study offers additional insights into the roles played by adaptive and maladaptive forms of humor.

These results suggest use of self-oriented humor may be a manifestation of one's relationship to one's self, similar to the concept of self-esteem. Higher use of selfenhancing humor may indicate a better relationship with one's self, while self-defeating humor may indicate a worse relationship with one's self. Although humor style and selfesteem are weakly correlated with one another, they are likely different constructs (Stieger et al., 2011).

\section{Limitations}

While the results obtained in this study are exciting, it is important to keep in mind some limitations to this study. One of these limitations is in regard to the homogeneity of the sample. The second limitation is the descriptive nature of the study rather than experimental. The sample was limited to university students predominantly in their first year of study. As such, the sample has a low average age. Additionally, most of the participants identified themselves as Caucasian/White, and as coming from middleto high socio-economic status. While the sample was relatively representative of the population at this campus, it was limited to students who took introductory psychology during the course of one quarter.

In addition to a homogenous sample, the design in this study is correlational. Since this was not an experimental design, directionality of effect is impossible to 
determine. It is impossible to say, at this point in time, if it is humor style that determines quality of life or if quality of life determines humor style.

\section{Future Directions}

Despite the limitations mentioned previously, the results obtained in this study are exciting. Further research regarding the influence of culture on humor style is still needed, e.g., humor styles in individuals with different sexual orientation, ability, age, and social status. Since this sample was homogenous, future research should explore how well humor styles are able to predict quality of life in cultures not represented here, e.g., lower SES, age, and acquired disability.

Future research should also explore how humor styles manifest themselves in collectivistic cultures. Previous research has found the aggressive style of humor is less distinct in the collectivistic cultures of China and Lebanon, respectively (Chen \& Martin, 2007; Taher et al., 2008). In addition, Taher et al. (2008) found two items from the Humor Styles Questionnaire meant to assess maladaptive humor styles loaded more strongly on their adaptive humor style counterparts. Together these suggest what is considered maladaptive in individualistic cultures may be less maladaptive or even not maladaptive in collectivistic cultures. Using the design employed in this study with a sample from a collectivistic society may yield different results where humor styles considered maladaptive by individualistic cultures might serve adaptive functions. Quality of life and its domains might also be significantly predicted by the other directed humor styles (Affiliative and Aggressive) more so than by the self-directed humor styles (Self-enhancing and Self-defeating). 
Along similar lines, future research might consider using more sophisticated statistical measures. This study assumed the relationship between humor styles and quality of life would be linear. It is possible there is a significant relationship between Affiliative and Aggressive humor styles, quality of life and its domains. Adaptive humor may be adaptive only to a point or in moderation. For example, using self-enhancing humor may be adaptive to a certain point, after which it fails to be functional (e.g., using self-enhancing humor to avoid dealing with reality). Future research should consider looking for a curvilinear relationship.

Beyond exploring how endorsement of particular humor styles may predict quality of life, future research should explore how humor styles can be manipulated to determine directionality. If direction can be established, there might be some stimulating prospects with regard to possible therapeutic interventions. Future research could examine the possibility of a humor skills training in an attempt to alter humor styles. Other research could explore the influence of psychotherapy on one's humor style.

In a pilot study, a group of researchers taught humor as a coping skill to a group of depressed people in an in-patient unit and found a significant increase in both state cheerfulness, trait cheerfulness, and coping humor (Falkenberg, Buchkremer, Bartels, \& Wild, 2011). According to Chen and Martin (2007) and Martin et al. (2003), coping humor is conceptually very similar to self-enhancing humor, and trait cheerfulness correlates positively with adaptive humor styles and negatively with maladaptive humor styles. Therefore, an intervention similar to that used by Falkenberg et al. (2011) might help increase the use of adaptive styles of humor and decrease maladaptive styles of humor and influence mental health. 


\section{Conclusion}

The purpose of this study was to determine whether or not quality of life and its domains could be significantly predicted by humor styles. It was hypothesized the adaptive styles of humor styles (affiliative and self-enhancing) would positively predict quality of life and its domains while maladaptive humor styles (aggressive and selfdefeating) would negatively predict quality of life and its domains. The data suggest there is partial support for the hypotheses previously outlined. This study was not without its limitations i.e., homogeneity of the sample and lack of an experimental design. Future studies can overcome these limitations by replicating this design using more culturally heterogeneous samples and exploring the possibility of altering humor style for the purpose of influencing quality of life. 


\section{References}

Adler, C. M., \& Hillhouse, J. J. (1996). Stress, health, and immunity: A review of the literature. In T. W. Miller (Ed.), Theory and assessment of stressful life events (pp. 109-138). Madison, CT: International Universities Press.

American Psychiatric Association. (2000). Diagnostic and statistical manual of mental disorders $\left(4^{\text {th }}\right.$ ed., text rev.). Washington, DC: Author.

Anderson, C. A., \& Arnoult, L. H. (1989). An examination of perceived control, humor, irrational beliefs, and positive stress as moderators of the relation between negative stress and health. Basic \& Applied Social Psychology, 10, 101-117. doi:10.1207/s15324834basp1002_1

Asthana, H. S. (2009). Quality of life: A psychological analysis. Psychological Studies, 54, 109-113. doi: 10. 1007/s12646-009-0017-2

Berger, A. (1987). Humor an introduction: Superiority theories, incongruity theories, psychoanalytic theories, cognitive theories of humor: Four theorists consider a joke. American Behavioral Scientist, 30, 6-15.

Berlyne, D. E. (1972). Humor and its kin. In J. H. Goldstein, \& P. E. McGhee (Eds.), The psychology of humor: Theoretical perspectives and empirical issues (pp. 43-60). New York: Academic Press.

Bruehl, S., Carlson, R., \& McCubbin, J. A. (1993). Two brief interventions for acute pain. Pain, 54, 29-36.

Cann, A., Norman, M. A., Wellbourne, J. L., \& Calhoun, L. G. (2008). Attachment styles, conflict styles, and humor styles: Interrelationships and associations with 
relationship satisfaction. European Journal of Personality, 22, 131-146. doi: 10.1002/per.666

Cann, A., Zapata, C. L., \& Davis, H. B. (2011). Humor style and relationship satisfaction in dating couples: Perceived versus self-reported humor styles as predictors of satisfaction. Humor: International Journal of Humor Research, 24, 1-20. doi: 10.1515/HUMOR.2011.001

Carbelo-Baquero, B. B., Alonso-Rodriguez, M. C., Valero-Garces, C. C., \& Thorson, J. A. (2006). A study of sense of humor in Spanish and American samples. North American Journal of Psychology, 8, 447-454.

Carroll, J. L., \& Schmidt, J. T. (1992). Correlation between humorous coping style and health. Psychological Reports, 70, 402. doi: 10.2466/PR0.70.2.402-402

Cattell, R. B., \& Luborsky, L. B. (1947). Personality factors in response to humor. Journal of Abnormal \& Social Psychology, 42, 402-421.

Cattell, R. B., \& Tollefson (1966). The IPAT humor test of personality. Champaign, IL: Institute of Personality and Ability Testing.

Chen, G., \& Martin, R. A. (2007). A comparison of humor styles, coping humor, and mental health between Chinese and Canadian university students. Humor: International Journal Of Humor Research, 20, 215-234. doi:10.1515/HUMOR.2007.011.

Cogan, R., Cogan, D., Waltz, W., \& McCue, M. (1987). Effects of laughter and relaxation on discomfort thresholds. Journal of Behavioral Medicine, 10, 139-144. doi:10.1007/BF00846422 
Cohen, J. (1988). Statistical power analyses for the behavioral sciences. (2nd edition). New Jersey: Lawrence.

Cohen, S., Frank, E., Doyle, W. J., Skoner, D. P., Rabin, B. S., \& Gwaltney, J. M. (1998). Types of stressors that increase susceptibility to the common cold in healthy adults. Health Psychology, 17, 214-223.

Cousins, N. (1979). Anatomy of an illness. New York: Norton.

Craik, K. H., Lampert, M. D., \& Nelson, A. J. (1993). Research Manual for the Humorous Behavior Q-sort Deck. Berkeley, CA: University of California, Institute of Personality and Social Research.

Craik, K. H., Lampert, M. D., \& Nelson, A. J. (1996). Sense of humor and styles of everyday humorous conduct. International Journal of Humor Research, 9, 273-302. doi:10.1515/humr.1996.9.3-4.273

Cummins, R. A., McCabe, M. P., Romeo, Y., \& Gullone, E. (1994). The Comprehensive Quality of Life Scale (ComQol): Instrument development and psychometric evaluation on college staff and students. Educational And Psychological Measurement, 54, 372-382. doi:10.1177/0013164494054002011

Dantzer, R., \& Mormede, P. (1995). Psychoneuroimmunology of stress. In B. E. Leonard \& K. Miller (Eds.), Stress, the immune system and psychiatry (pp. 47-67). Chichester, England: Wiley.

Deckers, L. (1993). On the validity of a weight-judging paradigm for the study of humor. Humor: International Journal of Humor Research, 6, 43-56. 
Diener, E., \& Biswas-Diener, R. B. (2002). Will money increase subjective well-being? A literature review and guide to needed research. Social Indicators Research, 57, 119-169. doi:10.1023/A:1014411319119

Dillon, K. M., \& Totten, M. C. (1989). Psychological factors, immunocompetence, and health of breast-feeding mothers and their infants. Journal of Genetic Psychology, $150,155-162$.

Dillon, K. M., Minchoff, B., \& Baker, K. H. (1985). Positive emotional states and enhancement of the immune system. International Journal of Psychiatry in Medicine, $15,13-17$.

Dixon, N. F. (1980). Humor: A cognitive alternative to stress? In I. G. Sarason \& C. D. Spielberger (Eds.), Stress and Anxiety, Vol. 7 (pp. 281-289). Washington, DC: Hemisphere.

Dozois, D. A., Martin, R. A., \& Bieling, P. J. (2009). Early maladaptive schemas and adaptive/maladaptive styles of humor. Cognitive Therapy And Research, 33, 585-596. doi:10.1007/s10608-008-9223-9

Fabrizi, M. S., \& Pollio, H. R. (1987). A naturalistic study of humorous activity in a third, seventh, and eleventh grade classroom. Merrill-Palmer Quarterly, 33, 107-128.

Falkenberg, I., Buchkremer, G., Bartels, M., \& Wild, B. (2011). Implementation of a manual-based training of humor abilities in patients with depression: A pilot study. Psychiatry Research, 186, 454-457.

Fallowfield, L. (1990). The quality of life: The missing measurement in health care. Souvenir Press. 
Feingold, A., \& Mazzella, R. (1991). Psychometric intelligence and verbal humor ability. Personality and Individual Differences, 12, 427-435. doi:10.1016/0191-8869(91)90060-O

Feingold, A., \& Mazzella, R. (1993). Preliminary validation of a multidimensional model of wittiness. Journal of Personality, 61, 439-456.

Ferrans, C. (1996). Development of a conceptual model of quality of life. Scholarly Inquiry For Nursing Practice, 10, 293-304.

Fitts, S. D., Sebby, R. A., \& Zlokovich, M. S. (2009). Humor styles as mediators of the shyness-loneliness relationship. North American Journal of Psychology, 11, 257-272.

Fredrickson, B. L. (1998). What good are positive emotions? Review of General Psychology, 2, 300-319.

Freud, S. (1905/1960). Jokes and their relation to the unconscious. New York: Norton. Freud, S. (1928). Humour. International Journal of Psychoanalysis, 9, 1-6.

Frewen, P. A., Brinker, J., Martin, R. A., \& Dozois, D. A. (2008). Humor styles and personality-vulnerability to depression. Humor: International Journal of Humor Research, 21, 175-195. doi: 10.1515/HUMOR.2008.009

Friedman, H. S., Tucker, J. S., Tomlinson-Keasey, C., Schwartz, J. E., Wingard, D., Criqui, M. (1993). Does childhood personality predict longevity? Journal of Personality \& Social Psychology, 65, 176-185.

Fry, P. S. (1995). Perfectionism, humor, and optimism as moderators of health outcomes and determinants of coping styles of women executives. Genetic, Social, \& General Psychology Monographs, 121, 211-245. 
George, D., \& Mallery, P. (2003). SPSS for Windows step by step: A simple guide and reference. 11.0 update (4th ed.). Boston: Allyn \& Bacon.

Greengross, G., Martin, R. A., \& Miller, G. (2012). Personality traits, intelligence, humor styles, and humor production ability of professional stand-up comedians compared to college students. Psychology Of Aesthetics, Creativity, And The Arts, 6, 74-82. doi: $10.1037 / \mathrm{a} 0025774$

Guyton, A. C., \& Hall, J. E. (2005). Textbook of medical physiology. Philadelphia: Saunders.

Hall, J. A. (2010). Is it something I said? Sense of humor and partner embarrassment. Journal of Social and Personal Relationships, 28, 383-405. doi: 10.1177/026540710

Hampes, W. P. (2005). Correlations between humor styles and loneliness. Psychological Reports, 96, 747-750. doi: 10.2466/PR0.96.3.747-750

Hampes, W. P. (2006). Humor and shyness: The relation between humor styles and shyness. Humor: International Journal of Humor Research, 19, 179-187. doi: 10.1515/HUMOR.2006.009

Higginson, I. J., \& Carr, A. J. (2001). Measuring quality of life: Using quality of life measures in the clinical setting. BMJ: British Medical Journal, 322, 1297-1300. doi:10.1136/bmj.322.7297.1297

Hubert, W, Moeller, M., \& de Jong-Meyer, R. (1993). Film-induced amusement changes in saliva cortisol levels. Psychoneuroendocrinology, 18, 265-272.

Hudak, D. A., Dale, A., Hudak, M. A., \& DeGood, D. E. (1991). Effects of humorous stimuli and sense of humor on discomfort. Psychological Reports, 69, 779-786. doi: 10.2466/PR0.69.7.779-786 
Hugelshofer, D. S., Kwon, P., Reff, R. C., \& Olson, M. L. (2006). Humor's role in the relation between attributional style and dysphoria. European Journal of Personality, 20, 325-336. doi: 10.1002/per.586

Janes, L.M. , \& Olson, J. M.(2000). Jeer pressure: The behavioral effects of observing ridicule of others. Personality and Social Psychology Bulletin, 26, 474-485.

Karkkanen, P., Kuiper, N. A., \& Martin, R. A. (2004). Sense of humor, physical health, and well-being at work: A three-year longitudinal study of Finnish police officers. Humor: International Journal of Humor, 17, 21-35.

Kazarian, S. S., \& Martin, R. A. (2004). Humour styles, personality, and well-being among Lebanese university students. European Journal Of Personality, 18, 209-219. doi:10.1002/per.505.

Kazarian, S. S., \& Martin, R. A. (2006). Humor styles, culture-related personality, wellbeing, and family adjustment among Armenians in Lebanon. Humor: International Journal Of Humor Research, 19, 405-423. doi:10.1515/HUMOR.2006.020.

Kohler, G., \& Ruch, W. (1996). Sources of variance in current sense of humor inventories: How much substance, how much method variance?. Humor: International Journal of Humor Research, 9, 363-397. doi: 10.1515/humr.1996.9.34.363

Korotkov, D., \& Hannah, T. E. (1994). Extraversion and emotionality as proposed superordinate stress moderators: A prospective analysis. Personality and Individual Differences, 16, 787-792. doi: 10.1016/0191-8869(94)90220-8

Kubie, L. S. (1971). The destructive potential of humor in psychotherapy. American Journal of Psychiatry, 127, 37-42. 
Kuiper, N. A., \& Martin, R. A. (1998). Is sense of humor a positive personality characteristic?. In W. Ruch, W. Ruch (Eds.), The sense of humor: Explorations of a personality characteristic (pp. 159-178). Berlin Germany: Walter de Gruyter \& Co.

Kuiper, N. A., \& McHale, N. (2009). Humor styles as mediators between self-evaluative standards and psychological well-being. Journal of Psychology: Interdisciplinary and Applied, 143, 359-376. doi:10.3200/JRLP.143.4.359-376.

Kuiper, N. A., Martin, R. A., \& Dance, K. A. (1992). Sense of humour and enhanced quality of life. Personality And Individual Differences, 13, 1273-1283. doi:10.1016/0191-8869(92)90169-P.

Kuiper, N. A., Martin, R. A., \& Olinger, L. (1993). Coping humour, stress, and cognitive appraisals. Canadian Journal of Behavioural Science, 25, 81-96. doi:10.1037/h0078791.

Kuiper, N. A. \& Nicholl, S. (2004). Thoughts of feeling better? Sense of humor and physical health. Humor: International Journal of Health, 17, 37-66.

Labott, S. M., \& Martin, R. B. (1990). Emotional coping, age, and physical disorder. Behavioral Medicine, 16, 53-61.

Lambert, R. B., \& Lambert, N. K. (1995). The effects of humor on secretory Immunoglobulin-A levels in school-aged children. Pediatric Nursing, 21, 16-19.

Lefcourt, H. M. (2001). Humor: The psychology of living buoyantly. New York: Kluwer Academic Publishers.

Lefcourt, H. M., \& Martin, R. A. (1986). Humor and life stress: Antidote, to adversity. New York: Springer. 
Lefcourt, H. M., Davidson-Katz, K., \& Kueneman, K. (1990). Humor and immunesystem functioning. Humor: International Journal of Humor Research, 3, 305-321. doi: 10.1515/humr.1990.3.3.305

Lefcourt, H. M., Davidson, K., Prkachin, K. M., \& Mills, D. E. (1997). Humor as a stress moderator in the prediction of blood pressure obtained during five stressful tasks. Journal of Research in Personality, 31, 523-542. doi: 10.1006/jrpe.1997.2191

Lefcourt, H. M., Davidson, K., Shepherd, R., Phillips, M., Prkachin, K. M., \& Mills, D. E. (1995). Perspective-taking humor: Accounting for stress moderation. Journal of Social \& Clinical Psychology, 14, 373-391.

Levine, J. (1977). Humour as a form of therapy: Introduction to symposium. In A. J. Chapman \& H. C. Foot (Eds.), It's a funny thing, humour (pp. 127-137). Oxford: Pergamon Press.

Lippa, R. A. (2007). The preferred traits of mates in a cross-national study of heterosexual and homosexual men and women: An examination of biological and cultural influences. Archives Of Sexual Behavior, 36, 193-208. doi:10.1007/s10508006-9151-2.

Lodico, C. (1998, February). An investigation of the efficacy of a stress-management intervention that utilizes humor as a central aspect of the program. (Doctoral dissertation). Retrieved from Proquest.

Lonner, W. J., \& Berry, J. (1986). Field Methods in Cross-Cultural Research. New York: Sage 
Martin, R. A. (1996). The Situational Humor Response Questionnaire (SHRQ) and Coping Humor Scale (CHS): A decade of research findings. Humor: International Journal Of Humor Research, 9, 251-272. doi:10.1515/humr.1996.9.3-4.251.

Martin, R. A. (2001). Humor, laughter, and physical health: Methodological issues and research findings. Psychological Bulletin, 127, 504-519. doi:10.1037/00332909.127.4.504.

Martin, R. A. (2002). Is laughter the best medicine? Humor, laughter, and physical health. Current Directions In Psychological Science, 11, 216-220. doi:10.1111/14678721.00204.

Martin, R. A. (2004). Sense of humor and physical health: Theoretical issues, recent findings, and future directions. Humor: International Journal Of Humor Research, 17, 1-19. doi:10.1515/humr.2004.005.

Martin, R. A. (2007). The psychology of humor: An integrative approach. Amsterdam Netherlands: Elsevier.

Martin, R. A., \& Kuiper, N. A. (1999). Daily occurence of laughter: Relationships with age, gender, and Type A personality. Humor: International Journal of Humor Research, 12, 355-384. doi: 10.1515/humr.1999.12.4.355

Martin, R. A., Kuiper, N. A., Olinger, L., \& Dance, K. A. (1993). Humor, coping with stress, self-concept, and psychological well-being. Humor: International Journal of Humor Research, 6, 89-104. doi:10.1515/humr.1993.6.1.89.

Martin, R. A., \& Lefcourt, H. M. (1983). Sense of humor as a moderator of the relation between stressors and moods. Journal of Social and Personality Psychology, 45, 1313-1324. doi:10.1037/0022-3514.45.6.1313 
Martin, R. A., \& Lefcourt, H. M. (1984). Situational Humor Response Questionnaire: Quantitative measure of sense of humor. Journal Of Personality And Social Psychology, 47, 145-155. doi:10.1037/0022-3514.47.1.145

Martin, R. A., Lastuk, J. M., Jeffery, J., Vernon, P. A., \& Veselka, L. (2012). Relationships between the dark triad and humor styles: A replication and extension. Personality And Individual Differences, 52, 178-182. doi:10.1016/j.paid.2011.10.010

Martin, R. A., Puhlik-Doris, P., Larsen, G., Gray, J., \& Weir, K. (2003). Individual differences in uses of humor and their relation to psychological well-being: Development of the Humor Styles Questionnaire. Journal Of Research In Personality, 37, 48-75. doi:10.1016/S0092-6566(02)00534-2.

McClelland, D. C., \& Cheriff, A. D. (1997). The immunoenhancing effects of humor on secretory IgA and resistance to respiratory infections. Psychology and Health, 12, 329-344. doi: 10.1080/08870449708406711

Mindess, H., Miller, C., Turek, J., Bender, A., \& Corbin, S. (1985). The Antioch humor test: Making sense of Humor. New York: Avon Books.

Monro, D. (1967). Humor. In P. Edwards (Ed.) The Encyclopedia of Philosophy. New York: The Macmillan Company.

Mulkay, M. (1988). On humor: Its nature and its place in modern society. New York: Basil Blackwell.

Myers, D. G. (2000). The funds, friends, and faith of happy people. American Psychologist, 55, 56-67. doi:10.1037/0003-066X.55.1.56

Nerhardt, G. (1976). Incongruity and funniness: Towards a new descriptive model. In A. J. Chapman, H. C. Foot, A. J. Chapman, H. C. Foot (Eds.), Humor and laughter: 
Theory, research, and applications (pp. 55-62). Piscataway, NJ US: Transaction Publishers.

Nevo, O., Keinan, G., \& Teshimovsky-Arditi. (1993). Humor and pain tolerance. Humor: International Journal of Humor Research, 6, 71-88. doi: 10.1515/humr.1993.6.1.71

Oring, E. (1994). Humor and the suppression of sentiment. Humor: International Journal of Humor Research, 7, 7-26. doi:10.1515/humr.1994.7.1.7

Orth-Gomer, K. (1994). International epidemiological evidence for a relationship between social support and cardiovascular disease. In S. A. Shumaker \& S. M. Czajkowski (Eds.), Social support and cardiovascular disease (pp. 97-117). New York: Plenum.

Paulhus, D. L., \& Williams, K. M. (2002). The Dark Triad of personality: Narcissism, Machiavellianism and psychopathy. Journal Of Research In Personality, 36, 556563. doi:10.1016/S0092-6566(02)00505-6

Pincus, A. L., \& Lukowitsky, M. R. (2010). Pathological narcissism and narcissistic personality disorder. Annual Review of Clinical Psychology, 6, 421-446. doi:10.1146/annurev.clinpsy.121208.131215

Porterfield, A. L. (1987). Does sense of humor moderate the impact of life stress on psychological and physical well-being?. Journal of Research in Personality, 21, 306317. doi: 10.1016/0092-6566(87)90013-4

Robinson, V. (1983). Humor and health. In P. McGhee \& H. Goldstein (Eds.), Handbook of humor research.(pp. 157-168). New York: Springer-Verlag. 
Romero, E. J., Alsua, C. J., Hinrichs, K. T., \& Pearson, T. R. (2007). Regional humor differences in the United States: Implications for management. Humor: International Journal of Humor Research, 20, 189-201. doi: 10.1515/HUMOR.2007.009

Rothbart, M. K. (1973). Laughter in young children. Psychological Bulletin, 80, 247-256. doi:10.1037/h0034846.

Rotton, J. (1992). Trait humor and longevity: Do comics have the last laugh?. Health Psychology, 11, 262-266. doi: 10.1037/0278-6133.11.4.262

Ruch, W. (1992). Assessment of appreciation of humor: Studies with the 3WD humor test. In C. D. Spielberger \& J. N. Butcher (Eds.), Advances in Personality Assessment (Vol. 9, pp. 27-75). Hillsdale, NJ: Erlbaum.

Ruch, W. (1997). State and trait cheerfulness and the induction of exhilaration: A FACS study. European Psychologist, 2, 328-341. doi:10.1027/1016-9040.2.4.328

Ruch, W. (Ed.). (1998). The sense of humor: Explorations of a personality characteristic. Berlin Germany: Walter de Gruyter \& Co.

Ruch, W., \& Hehl, F.J. (1998). A two-mode model of humor appreciation: Its relation to aesthetic appreciation and simplicity complexity of personality. In W. Ruch (Ed.), The sense of humor: Explorations of personality characteristic (pp.109-142). New York: Moutonde Gruyter.

Ruch, W., \& Kohler, G. (1998). A temperament approach to humor. In W. Ruch (Ed.), The sense of humor: Explorations of a personality characteristic (pp. 203-230). New York: Moutonde Gruyter. 
Ruch, W., \& Kohler, G. (1999). The measurement of state and trait cheerfulness. In I. Mervielde, I. J. Deary, F. De Fruyt, \& F. Ostendorf (Eds.), Personality psychology in Europe (pp. 67-83). Tilburg, the Netherlands: Tilburg University Press.

Saroglou, V. (2004). Being religious implies being different in humor: Evidence from self- and peer-ratings. Mental Health, Religion, \& Culture, 7, 255-267.

Saroglou, V. S., \& Scariot, C. (2002). Humor styles questionnaire: Personality and educational correlates in Belgian high school and college students. European Journal Of Personality, 16, 43-54. doi: 10.1002/per.430

Simon, J. M. (1990). Humor and its relationship to perceived health, life satisfaction, and morale in older adults. Issues in Mental Health Nursing, 11, 17-31. doi: $10.3109 / 01612849009014542$

Skevington, S. M., Lotfy, M. M., \& O'Connell, K. A. (2004). The World Health Organization's WHOQOL-BREF quality of life assessment: Psychometric properties and results of the international field trial. A Report from the WHOQOL Group. Quality Of Life Research: An International Journal Of Quality Of Life Aspects Of Treatment, Care \& Rehabilitation, 13, 299-310. doi:10.1023/B:QURE.0000018486.91360.00

Stieger, S., Formann, A. K., \& Burger, C. (2011). Humor styles and their relationship to explicit and implicit self-esteem. Personality And Individual Differences, 50, 747750. doi:10.1016/j.paid.2010.11.025.

Suls, J. (1972). A two-stage model for appreciation of joke and cartoons: An information processing analysis. In P. E. McGhee \& H. Goldstein (Eds.), The psychology of humor. New York: Academic. 
Suls, J. (1983). Cognitive processes in humor appreciation. In P. E. McGhee \& H. Goldstein (Eds.), Handbook of humor research. New York: Springer-Verlag.

Svebak, S. (1996). The development of the sense of humor questionnaire: From SHQ to SHQ-6. Humor: International Journal of Humor Research, 9, 341-361.

Taher, D., Kazarian, S. S., \& Martin, R. A. (2008). Validation of the Arabic Humor Styles Questionnaire in a community sample of Lebanese in Lebanon. Journal Of Cross-Cultural Psychology, 39, 552-564. doi:10.1177/0022022108321177.

Thorson, J.A., \& Powell, F.C. (1991). Measurement of sense of humor. Psychological Reports, 69, 691-701. doi:10.2466/PR0.69.8.1111-1115 (American Psychiatric Association, 2000)

Thorson, J. A., \& Powell, F. C. (1993a). Development and validation of the multidimensional sense of humor scale. Journal of Clinical Psychology, 49, 13-23. doi:10.1002/1097-4679(199301)49:1<13::AID-JCLP2270490103>3.0.CO;2-S.

Thorson, J. A., \& Powell, F. C. (1993b). Sense of humor and dimensions of personality. Journal of Clinical Psychology, 49, 799-809. doi:10.1002/10974679(199311)49:6<799::AID-JCLP2270490607>3.0.CO;2-P.

Thorson, J. A., Brdar, I., \& Powell, F. C. (1997). Factor-analytic study of sense of humor in Croatia and the USA. Psychological Reports, 81, 971-977.

Thorson, J. A., Powell, F. C., \& Samuel, V. T. (2001). Sense of humor in black and white. North American Journal of Psychology, 3, 1-12.

Thorson, J. A., Powell, F. C., Sarmany-Schuller, I., \& Hampes, W. P. (1997). Psychological health and sense of humor. Journal Of Clinical Psychology, 53, 605619. doi:10.1002/(SICI)1097-4679(199710)53:6<605::AID-JCLP9>3.0.CO;2-I. 
Tümkaya, S. (2007). Burnout and humor relationship among university lecturers. Humor: International Journal Of Humor Research, 20, 73-92. doi:10.1515/HUMOR.2007.004

Vaillant, G. E. (1977). Adaptation of life. Boston: Little Brown \& Co.

Vaillant, G. E. (2000). Adaptive mental mechanisms: Their role in a positive psychology. American Psychologist, 55, 89-98. doi:10.1037/0003-066X.55.1.89

Valdimarsdottir, H. B., \& Bovbjerg, D. H. (1997). Positive and negative mood: Association with natural killer cell activity. Psychology and Health, 12,319-327.

Vernon, P. A., Martin, R. A., Schermer, J., \& Mackie, A. (2008). A behavioral genetic investigation of humor styles and their correlations with the big-5 personality dimensions. Personality And Individual Differences, 44, 1116-1125. doi:10.1016/j.paid.2007.11.003

Veselka, L., Schermer, J. A., Martin, R. D., \& Vernon, P. A. (2010a). Laughter and resiliency: A behavioral genetic study of humor styles and mental toughness. Twin Research and Human Genetics, 13, 442-449.

Veselka, L., Schermer, J., Martin, R. A., \& Vernon, P. A. (2010b). Relations between humor styles and the Dark Triad traits of personality. Personality And Individual Difference, 48, 772-774. doi: 10.1016/j.paid.2010.01.017

Watson, D., \& Pennebaker, J. W. (1989). Health complaints, stress, and distress: Exploring the central role of negative affectivity. Psychological Review, 96, 234-254. doi: 10.1037/0033-295X.96.2.234

White, S., \& Camarena, P. (1989). Laughter as a stress reducer in small groups. Humor: International Journal of Humor Research, 2, 73-79. doi: 10.1515/humr.1989.2.1.73 
WHOQoL Group. (1998). Development of the World Health Organisation WHOQoLBREF quality of life assessment. Psychological Medicine, 28, 551-558.1998-0297000610.1017/S0033291798006667. 10.1017/S0033291798006667

Wojtyna, E., \& Stawiarska, P. (2009). Humor styles and psychosocial working conditions in relation to occupational burnout among doctors. Polish Psychological Bulletin, 40, 20-28. doi: 10.2478/s10059-009-0004-4

World Health Organization. (1991). World health statistics annual. Geneva: WHO.

Yip, J. A., \& Martin, R. A. (2006). Sense of humor, emotional intelligence, and social competence. Journal of Research in Personality, 40, 1202-1208. doi: 10.1016/j.jrp.2005.08.005

Yue, X., Hao, X., \& Goldman, G. (2010). Humor styles, dispositional optimism, and mental health among undergraduates in Hong Kong and China. Journal of Psychology in Chinese Societies, 11, 173-188.

Zeigler-Hill, V., \& Besser, A. (2011). Humor style mediates the association between pathological narcissism and self-esteem. Personality and Individual Differences, 50, 1196-1201. doi: 10.1016/j.paid.2011.02.006

Zelinsky, W. (1992). The Cultural Geography of the United States. Englewood Cliffs, NJ: Prentice-Hall.

Zillman, D. (1983). Disparagement humor. In P. E. McGhee \& J. H. Goldstein (Eds.), Handbook of humor research, Vol. 1 (pp. 85-108). New York: Springer.

Zweyer, K., Velker, B., \& Ruch, W. (2004). Do cheerfulness, exhilaration, and humor production moderate pain tolerance? A FACS study. Humor: International Journal of Humor Research, 17, 85-119. 